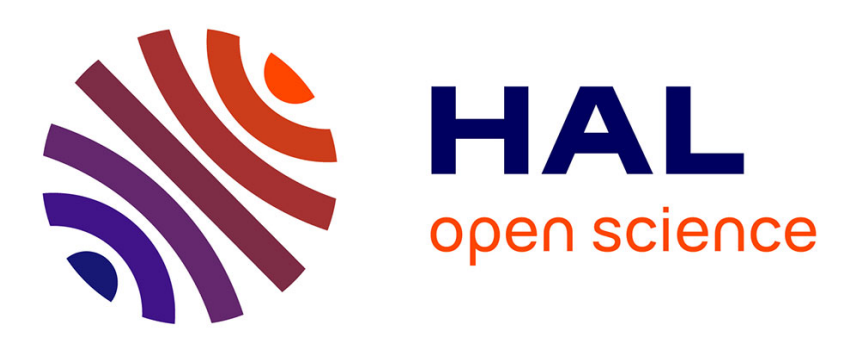

\title{
Controlling dislocation nucleation-mediated plasticity in nanostructures via surface modification
}

Jungho Shin, Lisa y Chen, Umut T Sanli, Gunther Richter, Stéphane Labat, Marie-Ingrid Richard, Thomas W. Cornelius, Olivier Thomas, Daniel Gianola

\section{- To cite this version:}

Jungho Shin, Lisa y Chen, Umut T Sanli, Gunther Richter, Stéphane Labat, et al.. Controlling dislocation nucleation-mediated plasticity in nanostructures via surface modification. Acta Materialia, 2019, 166, pp.572-586. 10.1016/j.actamat.2018.12.048 . hal-02002310

\author{
HAL Id: hal-02002310 \\ https://hal.science/hal-02002310
}

Submitted on 31 Jan 2019

HAL is a multi-disciplinary open access archive for the deposit and dissemination of scientific research documents, whether they are published or not. The documents may come from teaching and research institutions in France or abroad, or from public or private research centers.
L'archive ouverte pluridisciplinaire HAL, est destinée au dépôt et à la diffusion de documents scientifiques de niveau recherche, publiés ou non, émanant des établissements d'enseignement et de recherche français ou étrangers, des laboratoires publics ou privés. 


\section{Accepted Manuscript}

Controlling dislocation nucleation-mediated plasticity in nanostructures via surface modification

Jungho Shin, Lisa Y. Chen, Umut T. Sanli, Gunther Richter, Stéphane Labat, MarieIngrid Richard, Thomas Cornelius, Olivier Thomas, Daniel S. Gianola

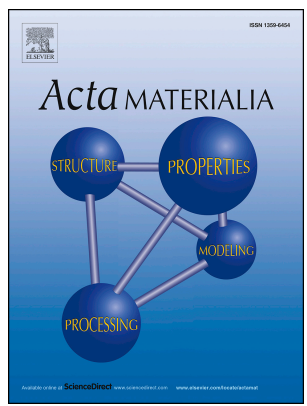

PII:

S1359-6454(18)30999-6

DOI:

https://doi.org/10.1016/j.actamat.2018.12.048

Reference: $\quad$ AM 15056

To appear in: Acta Materialia

Received Date: 18 September 2018

Revised Date: 11 December 2018

Accepted Date: 25 December 2018

Please cite this article as: J. Shin, L.Y. Chen, U.T. Sanli, G. Richter, S. Labat, M.-I. Richard, T. Cornelius, O. Thomas, D.S. Gianola, Controlling Dislocation Nucleation-Mediated Plasticity in Nanostructures via Surface Modification, Acta Materialia, https://doi.org/10.1016/j.actamat.2018.12.048.

This is a PDF file of an unedited manuscript that has been accepted for publication. As a service to our customers we are providing this early version of the manuscript. The manuscript will undergo copyediting, typesetting, and review of the resulting proof before it is published in its final form. Please note that during the production process errors may be discovered which could affect the content, and all legal disclaimers that apply to the journal pertain. 


\title{
Controlling Dislocation Nucleation-Mediated Plasticity in
}

\section{Nanostructures via Surface Modification}

\author{
Jungho Shin ${ }^{\text {a,b,c }}$, Lisa Y. Chen ${ }^{\text {a }}$, Umut T. Sanli ${ }^{\mathrm{d}}$, Gunther Richter ${ }^{\mathrm{d}}$, Stéphane Labat ${ }^{\mathrm{b}}$, Marie- \\ Ingrid Richard ${ }^{\text {be }}$, Thomas Cornelius ${ }^{\text {b }}$, Olivier Thomas ${ }^{\text {b }}$, Daniel S. Gianola ${ }^{\text {c,* }}$ \\ ${ }^{a}$ University of Pennsylvania, Philadelphia, PA, USA \\ ${ }^{\mathrm{b}}$ Aix Marseille Univ, Université de Toulon, CNRS, IM2NP, Marseille, France \\ ${ }^{\mathrm{C}}$ University of California - Santa Barbara, Santa Barbara, CA, USA \\ ${ }^{\mathrm{d}}$ Max Planck Institute for Intelligent Systems, Heisenbergstrasse 3, 70569 Stuttgart, Germany \\ ${ }^{\mathrm{e}}$ European Synchrotron Radiation Facility (ESRF), 6 rue Jules Horowitz, BP 220, F-38043 Grenoble \\ Cedex, France
}

\begin{abstract}
A straightforward strategy for reaching the ultimate strength of crystalline metals is to synthesize materials with a scarcity of defects. Bottom-up synthesis of nanostructures is an ideal means of approaching this limit, where nucleation of dislocations is a requirement in otherwise perfect crystals to facilitate plastic flow and mitigate brittle fracture. In this deformation mechanism regime where thermal fluctuations assist in the nucleation process, surface self-diffusion emerges as the rate-limiting step needed to promote displacive activity. We conducted tensile experiments in situ on pristine $\mathrm{Au}$ nanowires in addition to nanowires with thin $\mathrm{Al}_{2} \mathrm{O}_{3}$ coatings produced by atomic layer deposition. Distributions of yield strengths of nanowires with fully conformal coatings show a pronounced reduction in scatter and a measurable shift toward higher values. The presence of coatings did not curtail the ability of the nanowires to sustain significant amounts of plastic strain. In all conditions, nucleation of partial dislocations governed incipient plasticity. Analyses of the data using a thermal activation model in tandem with Bragg coherent X-ray diffraction point to the potent effect of control over the near surface zone in mediating diffusion, and thereby dislocation nucleation and the ensuing plastic response of the nanostructures.
\end{abstract}




\section{Introduction}

Crystalline nanowires (NWs) are candidates for next generation building blocks in novel devices due to their exceptional material properties [1-7]. Mechanical robustness is often a requirement in many applications of NWs given the extreme conditions that these materials experience during device operation. These NW systems often show a scarcity of defects owing to the bottom-up nature of synthesis, which thereby require nucleation of fresh dislocation content in order to accommodate plastic deformation [8-10]. In this mechanism regime, the theoretical strength can be achieved [11-15]. At the same time, undercoordinated atoms at the copious surfaces of these NWs exhibit a different material response compared to those in the interior, and can ultimately govern the collective properties [16-20]. These two distinctive features lead to unique material behavior in NWs; however, controlling their properties to sufficiently predict and tailor the mechanical properties of such materials via control of the dislocation nucleation process is still in its relative infancy.

In most bulk crystalline materials, the onset of plasticity relies on the glide of pre-existing dislocations, which are statistically stored inside the material. Various classical strengthening techniques were developed to impede this glide mechanism and thus tailor the material's strength [21-23]. However, as the characteristic length of a material approaches that of the intrinsic spatial distribution of pre-existing defects, the strength depends less on defect motion and relies more on dislocation nucleation to accommodate plasticity [24-26]. Size-dependent strengthening has drawn significant attention through experimentation on confined volumes in various crystal structure types [8]. While, the intrinsic size effect has to be interpreted carefully due to the role of modified material surfaces from focused ion beam (FIB) specimen preparation [25,27,28], strong size effects are present when some amount of dislocation content pre-exists in the material. 
On the other hand, a weak size dependence in strength has been reported from mechanical testing of defect free samples, fabricated either through a combination of directional solidification and selective etching [27,29] or near equilibrium growth via ultra-high vacuum physical vapor deposition approaches [15]. These NWs evidently require the nucleation of dislocations to undergo plastic deformation. However, dislocation nucleation is a thermally activated process, meaning that the strength is highly temperature dependent [30-34]. As a consequence, material strength distributions measured at finite temperatures manifest pronounced scatter due to the probabilistic nature of overcoming the nucleation barrier [34]. These two facts lead to an important question; how can we profit from the ultra-high strength of nucleation-controlled materials while managing the stochastic nature of plasticity in this regime?

The surface is expected to play a dominant role in mediating dislocation nucleation in defect-scarce nanostructures, where the large ratio of surface atoms to interior ones can be nonnegligible [30,35-38]. For instance, near-surface layers influenced by the undercoordinated atoms exhibit surface stresses that can influence the state of the bulk [35,39-41]. This effect can be significant for critical dimensions below $10 \mathrm{~nm}$, where counter balancing bulk stresses have been calculated to reach as high as $1 \mathrm{GPa}$ [42]. In addition to its impact on elastic properties, the surface is directly related to the activation of dislocation nucleation [43], whereby embryonic dislocation line length is reduced through the heterogeneous nucleation process $[42,44,45]$. Existing continuum models including contributions from dislocation line energy, stress dependent generalized stacking fault energy (GSF), image stresses, and surface ledge formation energy, predict corners where crystallographic facets meet to be the favorable nucleation site in nanowires under uniaxial deformation [46]. Preferential nucleation at corners has also been predicted by chain-of-states and free end nudged elastic band methods $[31,42,46]$. In most face 
centered cubic (FCC) NWs, Shockley partial dislocations are observed to nucleate from the surface as the incipient process and subsequently sweep through the material, leaving a residual stable stacking fault [47-49]. Despite the strong theoretical basis for the energetics of surface nucleation, a comprehensive physical description of how the dislocation first nucleates from the surface has not been fully addressed. Consequentially, robust strategies for controlling the properties of such nucleation-controlled materials have yet to be realized.

Related studies on thermal activation parameters and nucleation strength have been intensively carried out to glean insights on the nucleation event $[31,33,45,50-53]$. The activation energy and volume characterize the probabilistic nature of dislocation nucleation owing to thermal fluctuations when the applied stress is below the elastic limit (the athermal strength). Modeling of indentation on single crystal Pt extracted an activation energy of $\sim 0.3 \mathrm{eV}$ and an activation volume similar to $\sim 1 b^{3}$, where $b$ is the Burgers vector, which is consistent with a heterogeneous nucleation event. However, extracting the activation parameters for free standing nanostructures has been mainly performed by atomistic simulations, due to the experimental challenges in directly observing the deformation mechanism or obtaining sufficient measurements for statistical analysis.

In simulations, the activation energy is often calculated by finding the maxima of the minimum energy path from the initial to the final state at high strain rate $\left(\sim 10^{8} \mathrm{~s}^{-1}\right)$. Subsequently, the laboratory scale nucleation strength is predicted through implementing transition state theory (TST) $[31,46,52,54,55]$. Despite the accuracy of finding the nucleation site and mechanisms, these results rely heavily on the applied atomic potentials or the way the models find the energy pathways, and do not account for long range surface diffusion activities, partly due to computational cost. However, it has been pointed out that diffusion can compete with displacive 
mechanisms for plastic deformation of nano-scale materials, even at room temperature [56]. In situ bending of ZnO NWs in transmission electron microscopy (TEM) showed anelastic recovery within several minutes owing to diffusional activity [36]. Moreover, the importance of surface diffusion is reported in several recent mechanical tests on nano-scale materials. Qualitative observations from in situ TEM indentation on Ag nanoparticles demonstrated liquid-like behavior in addition to pseudoelasticity [37]. Temperature dependent tensile tests on defectscarce Pd NWs have extracted an activation energy for dislocation nucleation comparable to the surface self-diffusion energy barrier as well as an atomic level activation volume [34]. Surface diffusion activated by dislocation slip promoting uniform plastic deformation was directly observed during in situ TEM tensile testing of Ag NWs [38]. These results clearly demonstrate the interplay between surface diffusional activity and dislocation nucleation as carriers of plastic flow. In particular, the latter two works reported the strength of tensile tested NWs to approach their ideal strength. Hence, investigating means to control surface diffusion in nanomaterials may be a pathway for tailoring and predicting strength at the upper limit.

In this work, we present a strategy for tailoring the nucleation-controlled strength in metallic NWs without compromising tensile ductility. Au NWs were coated with ultra-thin layers of $\mathrm{Al}_{2} \mathrm{O}_{3}$ via atomic layer deposition (ALD) to alter the surface-mediated nucleation process. The coating layer is shown to impede surface diffusion, resulting in an increase of both the dislocation nucleation activation energy and activation volume, thereby strengthening the material while simultaneously reducing the scatter in the strength distribution.

\section{Experimental materials and methods}

\subsection{Sample preparation}


Single-crystalline $<110\rangle$ oriented Au NWs were grown by physical vapor deposition onto tungsten substrates using molecular beam epitaxy. The NWs were grown in an ultrahigh vacuum chamber in near equilibrium conditions, which lead to high purity crystals possessing atomically smooth and faceted surfaces, as described in detail in [15]. The grown wires were then divided into three groups for different surface coating conditions: uncoated, $3 \mathrm{~nm}$ thick coating, and 10 nm thick coating. The $\mathrm{Al}_{2} \mathrm{O}_{3}$ coatings were deposited using ALD. Bright field (BF) TEM images of uncoated and coated Au NWs are shown in Fig. 1a-1d. During the ALD process, the substrate temperature was maintained at $115^{\circ} \mathrm{C}(388.15 \mathrm{~K})$ with a chamber pressure of $12 \mathrm{~Pa}$. Nitrogen was used as the transport gas. Trimethylaluminum (TMA) and $\mathrm{H}_{2} \mathrm{O}$ were used as precursors: TMA was pulsed for $60 \mathrm{~ms}$ and then purged from the chamber for $10 \mathrm{~s}$, followed by a pulse of water for $60 \mathrm{~ms}$ and purging for $10 \mathrm{~s}$. The above process describes one cycle. Instead of bubbling the precursors, a "direct draw" method was employed, where $\mathrm{N}_{2}$ flowed through the lines, but not through the precursor bubbler, to carry the precursor. Nominal thicknesses of $3 \mathrm{~nm}$ and $10 \mathrm{~nm}$ ALD coatings were achieved by 40 cycles and 200 cycles, respectively (Fig. 1g); we use these nominal values throughout the article. ALD is expected to form an amorphous - and thus isotropic- layer with negligible misfit strains, which was confirmed by TEM in Fig. 1d and is in agreement with the literature [57,58]. Interdiffusion between the Au core and deposited $\mathrm{Al}_{2} \mathrm{O}_{3}$ was not observed.

We used a Kleindiek nanomanipulation system in a scanning electron microscope (SEM) to transfer individual NWs from the growth substrate to designated tensile stage setups, which are described in Section 2.2. A Pt-based gas injection system combined with electron beam induced deposition (EBID) served as the glue for harvesting and bonding of NWs onto the stage grips. The NWs on the growth substrate were prescreened to find optimum samples with minimal out 
of plane misorientation relative to the flat tensile stage. For this process, gentle contacts with the nanomanipulator tip were made at both the tip and the root of each NW to check the z-height difference. During the harvest step, the nanomanipulator tip was glued to the root side of the NW and mechanically pulled off of the substrate. In this way, plastic deformation prior to tensile testing could be avoided. The in-plane orientation of the harvested NW with respect to the tensile axis was adjusted by rotating the SEM stage that held the tensile setup. Finally, Pt-EBID was applied at the tensile grips to hold the sample in place. Often the NWs were intentionally mounted in a pre-buckled condition by pre-actuating the tensile stage to eliminate pre-strain during the clamping process.

\subsection{Tensile Testing}

Quantitative in situ tensile tests in the SEM were conducted using two types of tensile testing setups. The first type is a two-tower system consisting of a capacitance based load cell mounted on a 6 degree-of-freedom (DOF) positioner on one side and a linear piezo actuator on the other side $[59,60]$. Multiple NWs were harvested and affixed on the finger of a TEM half grid in a cantilever geometry during the first step of the transfer process. The TEM grid with the NWs was then mounted on the actuator side. After fine alignments by the 6 DOF positioner, the cantilevered NWs were gripped with Pt-EBID to the load cell for tensile tests. This configuration allows for higher throughput by enabling multiple NW tensile tests in series within one pump cycle as well as less transfer time for each NW. In addition, the NWs are readily prepared for TEM observations both before and after tensile testing.

The second set-up is a microelectromechanical system (MEMS) based thermal actuation tensile stage [61], which enables us to perform not only in situ tensile test in SEM but also 
temperature controlled tensile testing inside a custom built cryostat due to its compact design and low thermal mass [62]. In this set-up, the NW is mounted across the actuator grip and the load cell grip in a single manipulation step. Actuation is performed by joule heating the actuator-side silicon beam structures. The load is calculated from the displacement of the load cell, which has a pre-calibrated stiffness determined by resonance frequency measurements of the compound beam structure. In cases where Pt-EBID markers could be deposited on the NWs and imaged during tensile testing, digital image correlation was employed to measure local strain. In other cases, the relative grip displacements were used to estimate nominal strain, which could incorporate the effects of grip compliance. Unless otherwise indicated (e.g. Section 3.2), we refer to strain values as nominal ones. While both setups could be used for room temperature tensile testing, the latter was used for temperature controlled tensile testing [62]. Liquid nitrogen and a resistive heating block were used for temperature control inside the chamber. These tensile tests were performed at nominal strain rates of $10^{-4} \mathrm{~s}^{-1}$ and below $10^{-2} \mathrm{~Pa}$ of vacuum.

\subsection{Bragg Coherent Diffraction for nanowire cross-section}

The cross-sectional shapes of several Au NWs were determined by Bragg coherent X-ray diffraction (BCD), which were performed either at the CRISTAL beamline at SOLEIL synchrotron or at the ID01 beamline at the European Synchrotron Radiation Facility (ESRF). At both beamlines the incident X-ray beam was focused using a Fresnel zone plate (FZP). Whereas at the CRISTAL beamline the $8.5 \mathrm{keV}$ incident beam was focused down to $2 \mu \mathrm{m}(\mathrm{V}) \times 2 \mu \mathrm{m}(\mathrm{H})$, the focal spot of the $8 \mathrm{keV} X$-ray beam at the ID01 beamline was $200 \mathrm{~nm}(\mathrm{~V}) \times 500 \mathrm{~nm}(\mathrm{H})$. At both beamlines, the coherent part of the incident X-ray beam was selected by closing slits installed in front of the FZP to match the lateral coherence lengths which amount to $20 \mu \mathrm{m}(\mathrm{H}) \mathrm{x}$ 
$60 \mu \mathrm{m}(\mathrm{V})$ at the CRISTAL beamline and $60 \mu \mathrm{m}(\mathrm{H}) \times 200 \mu \mathrm{m}(\mathrm{V})$ at the ID01 beamline. The diffracted X-rays were recorded by a 2D MAXIPIX detector with a pixel size of $55 \mu \mathrm{m} \times 55 \mu \mathrm{m}$ installed $1 \mathrm{~m}$ downstream from the sample similar to the set-up in Ref. [63]. The threedimensional intensity distribution in the vicinity of the Au 111 Bragg peak was recorded by rocking the sample by $+/-1^{\circ}$ (corresponding to $\Delta Q=+/-0.9 \mathrm{~nm}^{-1}$ ). Subsequently, the electron density of the examined NWs was reconstructed from the recorded $3 \mathrm{D}$ coherent diffraction patterns, thus representing the NW cross-sectional shape [64].

\section{Results and Discussion}

\subsection{Tensile response and deformation behavior}

We first describe the tensile behavior of the $<011>$ Au NWs with various surface treatments, which is demonstrated by representative stress-strain curves in Fig. 2. The uncoated Au NWs provide the baseline response, which includes data from Ref. [65] obtained from NWs synthesized under identical growth conditions as those from the current study. All stress-strain curves are characterized by a period of elastic loading, followed by a transition to plastic deformation noted by relatively smooth flow behavior that extended for several percent of strain. The absence of intermittent load drops in the flow regime is consistent with previous reports on defect-scarce Au NWs [47], where two categories of plastic response were identified: (i) smooth transition from yield to flow and slip traces that are spatially homogenous along the NW length, and (ii) large stress drops following yield and localized plasticity associated with twin nucleation and growth $[47,49,65,66]$. The stress-strain response of the $3 \mathrm{~nm}$ and $10 \mathrm{~nm}$ coated Au NWs are reminiscent of the former category, suggesting that plasticity distributes along the length of the NW, consistent with our in situ observations in SEM (Supplementary Movies 1 and 2). We note 
that one of the curves for the uncoated Au NWs in Fig. 2 exhibits an initially low slope due to the NW being pulled taught from a pre-buckled condition; thus, these strain values are nominal ones as described in Section 2.2. For this particular curve, limited plasticity is observed.

The presence of $3 \mathrm{~nm}$ ALD coatings appears to allow for the NWs to maintain their strength and some extent of plastic deformation relative to the uncoated NWs. The same is true for the 10 $\mathrm{nm}$ coatings once the influence of the thicker coating on load bearing capability is subtracted and the intrinsic response of the Au core is plotted (as shown in Fig. 2 and the composite model described in Section 3.2). Taken as a whole, the coated Au NWs studied in the present work demonstrate high flow stress following yield, and ultimately abrupt fracture without any noticeable softening. All NWs exhibited critical resolved yield stresses that ranged from $0.01 \mu$ to $0.04 \mu$, where $\mu$ is the shear modulus, consistent with estimates of the theoretical shear strength [67]. Fig. 2b-d show representative SEM micrographs of tensile fractured Au NWs. We observe very similar postmortem images between the three groups. The absence of a large portion of a reoriented configuration of the NW suggests that any nucleated deformation twins had not grown substantially along the NWs. As a whole, these deformation morphologies are consistent with the interpretation of our stress-strain curve behavior where plastic hardening can prevent a dominant twin variant from propagating along the wire, leading to a sudden stress drop. Close inspection of the fractured region (inset of Fig. 2b-d) for all three NW types show localized ductile-like fracture.

Post-mortem TEM micrographs displayed evidence of plasticity that was distributed spatially along the NWs during testing (Fig. 3). Based on the stress-strain curves presented in Fig. 2a, extended twins along the wire would not be expected, which was confirmed by the TEM micrographs. The uncoated NW (Fig. 3a) showed the appearance of multiple nanotwins 
separated by slip traces which formed during tension. The angle of the slip traces is consistent with $\{111\}$ planes. Given the $<011>$ Au NW loading in tension, where the Schmid factor for the leading partial dislocation is larger (0.471) than the trailing partial dislocation $(0.236)$, as well as the short slip distances to traverse the NW, a propensity for the formation of extended stacking faults exists, whereby an atomic step is formed on the surface after this partial slip event $[41,42,68]$. This atomic step becomes a facile nucleation site for subsequent nucleation, which could be an additional leading partial nucleated on an adjacent slip plane (twinning partial dislocation) leading to the nucleation of a $\Sigma\{111\}<10-1>$ deformation twin $[47,49,66]$. Another pathway could an additional leading partial dislocation nucleated at a different location; in all cases the incipient process is mediated by nucleation of partial dislocations. Close inspection of these deformed segments suggests a local reorientation of the crystal, as evidenced by nm-sized kinks on the surface owing to twin formation. A single variant was activated, based on the consistent angle between the slip traces along the deformed NW. For the $3 \mathrm{~nm}$ coated NW (Fig. 3b), we observed spatially distributed thin parallel slip traces corresponding to a single slip system along the wire, with the same projected slip trace angle as that found in the uncoated NW. Spatially extended twinned segments were not observed, suggesting either many nanotwin domains consisting of a few atomic planes, or extended stacking faults. The deformed $10 \mathrm{~nm}$ coated NW in Fig. 3c shows that two slip systems were activated along the wire during its tensile test, both with slip trace angles identified as equally probable $\{111\}$ planes. The slip traces were found to overlap each other, which may have resulted in dislocation jogs at the junctions. Apart from the fractured tip ends, the absence of distinct grain reorientation indicates the formation of multiple nanotwins or extended stacking faults along the NW like in the $3 \mathrm{~nm}$ coated NW. As a whole, all three $\mathrm{Au}$ NWs formed distinctive slip traces formed by a single equivalent variant that 
spatially distribute along the wire length, contributing to stable and sustained plastic deformation. The deformation mode in all three groups of $<011>\mathrm{Au}$ NWs in tension resides in a category reported in previous results [47], where multiple nanotwins are formed along the tensile tested $\mathrm{Au}$ NWs in contrast to the mode where one dominant twinned grain propagates all the way through the nanowire. Therefore, we conclude that (i) the coating does not alter the governing mechanism for incipient yielding based on surface nucleation of partial dislocations, and (ii) that these observations are consistent with tensile behavior demonstrating sustained plasticity after yielding and the suppression of immediate runaway localization.

Further examination of the slip traces in the $10 \mathrm{~nm}$ coated NWs was performed, as shown in Fig. $4 a-4 c$ for imaging conditions along different zone axes. The red guide lines on the NWs delineate a slip trace in the 2D projection. Note that the same NW is presented in Fig. $4 \mathrm{a}$ and $4 \mathrm{~b}$ but with different viewing angles, whereas $4 \mathrm{c}$ is a different NW. The slip traces are difficult to characterize when viewing along the [100] zone axis, whereas it is possible to distinguish two systems or variants when viewing along a [1-11] zone axis. Furthermore, the slip traces from [111] suggest that the NW has a truncated rhombic cross-sectional shape. From the [0-11] zone axis (Fig. 4c) the contrast of the slip trace becomes weak due to the edge-on view. The schematics in Fig. 4d illustrate different views of the most probable $\{111\}$ slip planes for partial dislocation nucleation that can be activated when these NWs are under tension along the $<01-1>$ growth axis. We confirmed that the slip traces observed in each TEM zone axis are consistent with the respective most probable crystallographic slip planes. Thus, regardless of the coating thickness, the presence of slip traces as observed in postmortem TEM (Fig. 3 and Fig. 4) suggests that partial dislocation mediated plasticity persists as the dominant deformation response via the creation of stacking faults and nanotwins. 
The locally necked region of the fractured NWs was examined in more detail as shown in Fig. 5. The fractured ends of all 3 types of NWs are cone-like in shape, characteristic of a ductile response, which also supports the conclusion that the fracture mechanism is independent of the presence of an $\mathrm{Al}_{2} \mathrm{O}_{3}$ coating. The dark field (DF) image of the circled diffraction spot in the inset of Fig. 5c is shown in Fig. 5d. The distinct reorientation of the fractured tip of the $10 \mathrm{~nm}$ coated NW indicates that deformation twinning via coordinated partial dislocation nucleation on adjacent slip planes is still a key mechanism underpinning plasticity in the coated NWs.

\subsection{Yield strength of the Au core}

The measured strength of a pristine FCC NW is determined by nucleation of the first partial dislocations from the surface as energetically preferred from a corner $[31,42,47,66]$, which is captured as the yield strength during tensile testing. In the case of the uncoated Au NWs, this strength can be calculated from the applied loads and the measured wire diameters. However, as the thickness of the coating increases, the volume fraction of the coating begins to contribute to load bearing on the composite system, which must be decoupled to determine the intrinsic response of the Au NW. Here, we calculate the Au core strength for coated NWs in order to deconvolute composite and surface modification effects. The directly measured NW yield strain by digital image correlation (DIC) gives the effective modulus, $E_{\text {eff, }}$ which is the elastic modulus of the entire composite from the elastic unloading portion of the stress-strain curves (see Fig. 6). From this measurement and using previously measured elastic moduli of Au NWs [65], the elastic modulus of the $10 \mathrm{~nm}$ thick $\mathrm{Al}_{2} \mathrm{O}_{3}$ coating $\left(E_{\text {coat }}\right)$ deposited at $115^{\circ} \mathrm{C}$ was calculated to be $122 \mathrm{GPa}$. Also shown in Fig. 6(b) is the fit to a smaller portion of the unloading curve, which gives a value of $E_{\text {coat }}=102 \mathrm{GPa}$ (albeit with a lower fit quality). While this range of values 
appears to be lower than reported values of $\mathrm{ALD} \mathrm{Al}_{2} \mathrm{O}_{3}(\sim 180 \mathrm{GPa})$ for thicker coatings deposited at over $150^{\circ} \mathrm{C}$ in thin film geometries and on non-metallic substrates [69-74], our measured values of $E_{\text {coat }}$ are consistent with previous studies when factoring in the dependencies

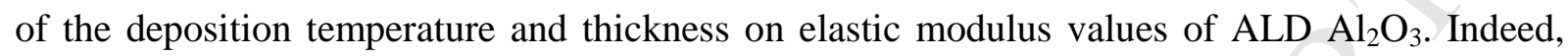
lower Young's moduli values in the range of approximately 125 to $150 \mathrm{GPa}$ are reported for growth temperatures below $150^{\circ} \mathrm{C}$, which is ascribed to lower alumina density and residual hydrogen content at lower deposition temperature $[75,76]$. In addition, thinner coatings such as those employed in our study generally correlate with lower densities [76]. We thus utilize the value of $E_{\text {coal }}=122 \mathrm{GPa}$ (corresponding to a more accurate fit in Fig. 6(b)) to deduce the yield strains obtained from an isostrain composite model for the remainder of the NWs and for subsequent analyses. This yield strain is then used to calculate the yield strength of the Au core, assuming the elastic modulus of $\mathrm{Au}$ along $\langle 011\rangle\left(E_{A u}\right)$. Surface stress effects are known to arise at small NW diameters, inducing compressive axial stresses as high as $1 \mathrm{GPa}$ for less than $10 \mathrm{~nm}$ in diameter. However, this compressive stress quickly diminishes for NWs with diameters such as ours (on the order of $100 \mathrm{~nm}$ ), which exhibit compressive stresses as low as approximately 10 MPa [42]. Thus, we expect negligible surface stress effects, which also has been experimentally shown in Ref. [65]. We also assume minor contributions from non-linear elasticity near the yield point in our estimates. The details of this procedure in this section can be found in Appendix A.

The corrected yield strengths of Au NW cores tested at room temperature as a function of the measured NW effective diameter is shown in Fig. 7. For all three groups of NWs, we see a large scatter in strength values at room temperature (ranging from 0.9 to $2.1 \mathrm{GPa}$ ) and a weak size dependence, similar to measurements on defect-scarce Pd NWs [34]. Note that the scatter band of the strength data is beyond the experimental uncertainty associated with the 
measurement. Details on these error calculations can be found in [34], but the two largest experimental uncertainties originate from the imaging resolution of diameter measurements $(<10 \%)$ and the assumption of an effective circular-cross sectional shape (between $10 \%$ and $21 \%$ depending on the azimuth viewing angle). Other factors such as the cumulative errors from variable strain rates and load-bearing of hydrocarbon-based contamination are calculated to be negligible [31,34]. Misalignment of the NW at the tensile stage is measured to be less than $3^{\circ}$, which contributes less than $50 \mathrm{~Pa}$ of bending stress at the grip end. The key notable result from Fig. 7 is that the overall scatter band of yield strengths remains large for the $3 \mathrm{~nm}$ coating relative to the uncoated data, but decreases for the thicker $10 \mathrm{~nm}$ coating. This result is insensitive to the choice of $E_{\text {coat }}$ obtained from our measurements to deduce core strengths for the $10 \mathrm{~nm}$ NWs, as demonstrated in the right panel of Fig. 7.

\section{Discussion}

\subsection{Room temperature strength distribution}

The key findings from our tensile tests of ALD coated Au NWs can be summarized as follows: the $\mathrm{ALD} \mathrm{Al}_{2} \mathrm{O}_{3}$ coatings influence the distributions of yield strength reflecting incipient plasticity, yet maintain high strength and the capacity for measurable plastic deformation, with partial dislocation nucleation-mediated plasticity of $\mathrm{Au}$ NWs persisting as the governing deformation mechanisms irrespective of the presence of the coatings. These results corroborate previously studied mechanisms on FCC NWs; namely, corners are the preferential nucleation site for partial dislocations, and the strength is determined by the first partial dislocation nucleation event $[31,42,47]$. Moreover, our statistical results on the strength of Au cores show a 
weak size dependence but a large scatter beyond the experimental uncertainty in the room temperature strength distribution, suggesting a thermally activated event governing the underlying deformation mechanism. Such a thermally activated nucleation process is described by TST, which predicts the probabilistic nucleation stress as a strong function of temperature and a weaker dependency on the sample size [31]. One of the most striking results is that the presence of $10 \mathrm{~nm}$ coatings serves to reduce the scatter band of yield strength distribution while shifting it toward large values. In this section, we discuss how the surface coatings serve to alter this thermal activation process by first comparing the strength distribution of room temperature tensile tests. For the following analysis, we adopt a model developed from TST to fit our experimental data and evaluate thermal activation parameters.

TST describes dislocation nucleation as a kinetic pathway from an initial to a final energy state in an energy landscape. The advancement passes through a minimum energy path by overcoming an energy barrier with a characteristic activation energy to form a stable dislocation loop $[31,34,45,51]$. While the athermal strength is then the theoretical elastic limit of a material in the absence of thermal energy, dislocations can probabilistically nucleate below this limit with the aid of thermal fluctuations when tested at finite temperature. At a given temperature and stress, the nucleation rate $v$ can be expressed as,

$\nu=N \nu_{0} \exp \left[\frac{\Delta G_{a c l}(\sigma, T)}{k_{b} T}\right]$

where $N$ is the number of equivalent nucleation sites, $v_{0}$ is the attempt frequency $k_{b}$ is the Boltzmann constant, and $\Delta G_{a c t}$ is the Gibbs free energy barrier to nucleate a dislocation. Noting that the stress can be expressed with Young's modulus, strain rate $(\dot{\epsilon})$ and time as $\sigma=E \dot{\epsilon} t$, an 
implicit form for the activation parameters can be obtained for a constant temperature $(T)$ and strain rate following $\mathrm{Zhu}$ et al. [31]:

$\frac{\Delta G_{a c t}(\sigma, T)}{k_{b} T}=\ln \frac{k_{b} T N \nu_{0}}{E \dot{\epsilon} \Omega(\sigma, T)}$.

This form is derived from $d f / d t=-v f$, which expresses the relationship between survival probability $f$ and nucleation rate $v[31,77]$. The activation volume $\Omega$ measures the stress sensitivity of the nucleation barrier, $\Omega(\sigma, T)=-\partial G /\left.\partial \sigma\right|_{T}$. Furthermore, the dependence of $\Delta G_{a c t}$ on stress and temperature can be expressed as,

$\Delta G_{a c t}(\sigma, T)=\Delta U_{a c t}\left(1-\frac{T}{T_{m}}\right)\left(1-\frac{\sigma}{\sigma_{a t h}}\right)^{\alpha}$

where $\Delta U_{\text {act }}$ is the energy barrier to nucleate a dislocation in the absence of external energy input, $T_{m}$ is a characteristic temperature, and $\sigma_{a t h}$ is the athermal strength. The exponent $\alpha$ is a stress dependent sensitivity factor, which is assumed to be 1 for constant activation volume [34,50], whereas values for $\alpha$ other than unity were deduced based on MD simulations of compression of Cu NWs $(\alpha=4.1)$ [31] and Mo nanoparticles $(\alpha=1.46)$ [53]. In a statistical analysis on Pd NWs, both $\alpha=1$ and $\alpha=4$ were considered to ascribe a stress dependency that appeared to vary with temperature [34]. While the activation volume can be stress dependent, we choose a linear dependence $(\alpha=1)$ for mathematical simplicity and a stress independent activation volume in order to compare the influence of the coating on the activation parameters. An expression for the cumulative distribution function (CDF), $F$ can be obtained from Eq. (2) and Eq. (3) as in Ref. [51], yielding 
$F(\sigma, T)=1-\exp \left\{\frac{k_{b} T N \nu_{0}}{E \dot{\epsilon} \Omega}\left[\exp \left(-\frac{\Delta U_{a c t}}{k_{b} T}\right)-\exp \left(\frac{\Omega \sigma-\Delta U_{a c t}}{k_{b} T}\right)\right]\right\}$

Subsequently, we performed nonlinear regression with Eq. (4) to the experimental cumulative probability distributions of NW strengths to obtain the activation parameters $\Delta U_{a c t}, \Omega$, and $N v_{0}$. The Levenberg-Marquardt nonlinear least squares algorithm was used for the fitting process with a maximum iteration number of 1500 and termination tolerance of $10^{-8}$ for the residual sum of squares.

The room temperature nucleation strength data and corresponding fits to the thermal activation model are overlaid in Fig. 8, shown as cumulative probability distributions. The uncoated data fit well to the model with a slight deviation at the high limit of the strength distribution. The $\Delta U_{a c t}$ value we extract for the uncoated NWs is $0.13 \mathrm{eV}$, which is significantly lower than the value predicted from atomistic simulations $(>1 \mathrm{eV})$ [31,55] due to possible atomic-scale flaws and their associated kinetics on the surface of real NWs. The activation volume $\Omega$ deduced from the fit is $0.71 b^{3}$, which compares to $1 \sim 10 b^{3}$ for heterogeneous nucleation reported from MD calculations [31]. We estimate a pre-exponential factor $\left(N v_{0}\right)$ of 0.3 $\mathrm{s}^{-1}$, which is clearly much lower than what would be estimated from a single nucleation site $(N=1)$ and with $v_{0}$ as the Debye frequency, and consistent with similar reports of discrepancies in attempt frequencies from other experimental studies [34,51].

Notably, our results show that the influence of the ALD coating on the activation parameters depends on the coating thickness. The strength distribution for the $3 \mathrm{~nm}$ coated NWs is slightly broader than that of the uncoated NWs, which is accompanied with a $4 \%$ decrease in $\Delta U_{a c t}$ and a $44 \%$ decrease in $\Omega$ with respect to the activation parameters for the uncoated NWs. Interestingly, the strength values at the high end of the distribution for the uncoated NWs overlap with the 3 
$\mathrm{nm}$ coated strength fit. In contrast, the $10 \mathrm{~nm}$ data shows that the spread of the strength distribution markedly decreases, suggesting a strong influence of the coating on the nucleation event. Compared to the uncoated NWs, both $\Delta U_{a c t}$ and $\Omega$ increase by $32 \%$ and $63 \%$ to values of $0.33 \mathrm{eV}$ and $6.01 b^{3}$, respectively, and the model is shown to fit the experimental data over the entire stress range. The fitted results for the room temperature experiments on all NWs are summarized in Table 1.

All three groups of NWs showed values of $\Delta U_{\text {act }}$ ranging from 0.25 to $0.33 \mathrm{eV}$. These values are substantially smaller than the values predicted from atomistic simulations for FCC crystals $[31,33,46]$, and which are comparable to the values associated with surface diffusional barriers

[78]. As further evidence, the activation volumes that we obtain are of the order of several $b_{p}{ }^{3}$ (corresponding to about $1 b^{3}$, where $b_{p}$ is the partial Burgers vector of Au). Previous MD simulations report $1 \sim 10 b^{3}$ for dislocation nucleation on atomically flat surface [31]. It has been shown that surface ledges or terraces can be stress amplifying sites, which may result in small activation volumes associated with the nucleation event [34]. The small activation energies and volumes that we measure imply a large thermal uncertainty, leading to highly probabilistic nucleation strengths, in contrast to the deterministic nature of bulk yield strengths (where $\Omega$ is $100 \sim 1000 b^{3}$ in FCC crystals). Strikingly, the $10 \mathrm{~nm}$ coating does serve to substantially narrow the nucleation distribution. The change in $N v_{0}$ for different coating thickness is relatively large among the three parameters; however, this amount of change is considered to be a higher order effect since the nucleation stress is only influenced by orders of magnitude changes in $N v_{0}$ [31].

\subsection{Temperature dependent strength of coated nanowires}


Before discussing the influence of the coating on dislocation nucleation, we further analyze the small values of activation energy and volume from the room temperature tests, which can be related to single atomic level activity. The implication of atomic scale nucleation events combined with the fact that the NWs have high surface to volume ratio suggests the role of surface diffusion as the most probable candidate for rate-limiting dislocation nucleation step at finite temperature. Experimental evidence on $\mathrm{Ag}$ and $\mathrm{Pd} \mathrm{NWs}$ corroborate this finding that surface diffusion plays a critical role in plastic deformation [34,38]. As explained previously, dislocations nucleate with the aid of surrounding thermal energy; thus, the strength is expected to be a strong function of temperature. To show further evidence, we investigated the temperature dependent strength of $10 \mathrm{~nm}$ coated Au NWs to elucidate how the coating can affect dislocation nucleation. We performed low temperature tests $(91 \mathrm{~K} \sim 171 \mathrm{~K})$ to quantify the temperature dependent nucleation strength distribution, which should collapse in the athermal limit.

Fig. 9a shows representative stress-strain curves of the $10 \mathrm{~nm}$ coated NWs tested at three different temperature $(91 \mathrm{~K}, 171 \mathrm{~K}$, and $295 \mathrm{~K})$. We clearly see a strong temperature dependence in the measured yield strength, although the shapes of the curves remain self-similar among the different test temperatures. Eq. (4) can be rearranged to express the temperature dependent behavior of strength as

$$
\sigma(T)=\frac{k_{b} T}{\Omega} \ln \left[\exp \left(-\frac{\Delta U_{a c t}}{k_{b} T}\right)-\frac{E \dot{\varepsilon}}{k_{b} T N \nu_{0}} \ln (1-F(\sigma))\right]+\frac{\Delta U_{a c t}}{\Omega} .
$$

Subsequently, Eq. (5) is fit to the temperature-controlled experimental strength including the room temperature tests to obtain the athermal strength value $\left(\sigma_{\text {ath }}=\Delta U_{\text {act }} / \Omega\right)$. Here, we assume a stress-independent activation volume $(\alpha=1)$, resulting in $\sigma_{\text {ath }}=2.26 \mathrm{GPa}, \Delta U_{\text {act }}=0.39 \mathrm{eV}$ and $\Omega=1.16 b_{p}{ }^{3}$. The resulting athermal strength value is comparable with the value of $2.5 \mathrm{GPa}$ 
estimated from atomistic simulations of a pristine Au NW with a diameter of $100 \mathrm{~nm}$ [46]. This result suggests that the coating may not affect the nucleation strength at $0 \mathrm{~K}$ where there is no thermal fluctuation, which is in line with our TEM observations showing a consistent deformation mechanism for uncoated and coated NWs (Fig. 3).

Fig. $9 \mathrm{~b}$ shows the temperature dependent strength data along with the model predictions of the probability distributions of dislocation nucleation. Vertical slices of the contour map represent normalized probability distribution functions (PDFs) of the nucleation stress at a given temperature, which are obtained from the derivative of the CDF with respect to stress in Eq. (5), normalized by the maximum value. The lower and the upper dotted lines represent 5 th and 95 th percentiles of the nucleation stress. For instance, the most probable nucleation stress at room temperature $\left(50^{\text {th }}\right.$ percentile at $\left.295 \mathrm{~K}\right)$ for the $10 \mathrm{~nm}$ coated NW is $1.28 \mathrm{GPa}\left(0.57 \sigma_{\text {ath }}\right)$. The PDF shows remarkable agreement with experimental data over the temperature range studied; indeed, the majority of the experimental strength data fall within this probability envelope.

We next estimate $\Delta G_{\text {act }}=\Delta U_{\text {act }}-\sigma_{\mathrm{c}} \Omega-\mathrm{T} \Delta S_{\text {act }}$ giving the finite temperature activation free energy evaluated at the most probable nucleation stress $\left(\sigma_{\mathrm{c}}\right)$. As a first order estimate, we assume negligible activation entropy to give $\Delta G_{\text {act }} \approx \Delta H_{\text {act }}=0.17 \mathrm{eV}$. This value is less than $1 / 3$ of the bulk vacancy migration energy of $0.71 \mathrm{eV}$ for $\mathrm{Au}[79]$ and less than $1 / 2$ of the vacancy migration energy at low index surfaces [80], which is well below the simulated activation energy values for dislocation nucleation (over $1 \mathrm{eV}$ ) [42]. Similarly, the activation Gibbs free energy calculated from temperature dependent tests on Pd NWs was reported to be much lower than the vacancy migration energy while being comparable to the activation energy of surface self-diffusion on $\{111\}$ surfaces, which are the most dominant facet of our system [34]. Our tested Au NWs have similar geometries as the Pd NWs where $\{111\}$ is the prevalent facet with $\langle 110\rangle$ as the growth 
direction. In the case of $\mathrm{Au}$, self-diffusion on $\{111\}$ was calculated by embedded atom methods using two different atomic potentials, giving barriers of $0.021 \mathrm{eV}$ (Adams, Foils, and Wolfer function) and $0.038 \mathrm{eV}$ (Voter and Chen function) [78]. In the same study, the surface selfdiffusion on $\{100\}$ surfaces were calculated to be $0.64 \mathrm{eV}$ (Adams, Foils, and Wolfer function) and $0.84 \mathrm{eV}$ (Voter and Chen function), showing a very large dependence on the surface crystallography.

We further refine our experimentally inferred values of $\Delta G_{\text {act }}$ by adopting a value of $\Delta S_{\text {act }}=$ $4.17 k_{b}$ based on values extracted from experiments on Pd NWs. This is rationalized by assuming a similar partial dislocation nucleation process in FCC $<110>$ NWs and similar faceting with $\{111\}$ and $\{100\}$ being the major and minor facets, respectively. Furthermore, the non-linearity parameter of $\mathrm{Pd}$ and $\mathrm{Au}$ is shown to be comparable, suggesting similar bond anharmonicity $[35,81]$. We thus estimate $\Delta G_{\text {act }}=0.063 \mathrm{eV}$ for the $10 \mathrm{~nm}$ coated Au NWs, which is of the order of the self-diffusion activation energy on $\{111\}$. Thus, the small activation volume that we obtain, coupled with low activation free energies implies that surface diffusional activity continues to be the rate-limiting step to dislocation nucleation, even in the coated NWs. Interestingly, the values we obtain demonstrate that the $10 \mathrm{~nm}$ coating serves to increase the activation barrier. In turn, diffusional activity is altered resulting in a higher strength at finite temperatures and a reduction in the probabilistic nature of yielding, while preserving ductility as shown in the previous sections.

\subsection{Role of nanowire faceting and coatings on surface diffusion}

The geometry and the crystallography of the NW has been shown in both simulation and experiments to be important in determining its mechanical behavior [65]. The strength varies by 
having distinct nucleation sites and can be rationalized by the dislocation line length difference arising from different cross-sectional shapes. For instance, MD simulations of $\mathrm{Cu}$ NW demonstrated a reduction of $3.5 \mathrm{GPa}$ of the yield strength for square nanowires compared to circular ones [68].

Up to this point, we have led our analysis with an assumed crystallographic shape of the NWs partly confirmed by electron microscopy images. In simulation studies, particularly <110> oriented NWs, the cross-sectional shapes have been chosen to be square, circular, rhombic, or truncated rhombic (quasi-hexagonal) $[41,46,47,68]$. However, in reality, the shape of $\langle 110\rangle$ oriented NWs grown on identical substrates can deviate from a truncated rhombic (hexagonal) to a rhombic shape (consisting of $\{111\}$ facets only), to even various ribbon shapes $[49,65,66,82]$. These experimentally observed shapes were primarily informed by interpreting $2 \mathrm{D}$ images, or alternatively, viewing the shape from the wire axis; however, the latter method requires destructive methods and may not provide the full surface crystallography. On the other hand, $\mathrm{BCD}$ allows for determining both the nanowire cross-section and crystalline orientation nondestructively and with a spatial resolution of $\sim 10 \mathrm{~nm}[63,83,84]$. For the following section, we review possible surface diffusion mechanisms that can occur on our experimentally determined geometry of the NW. Two adjacent facets meet at an edge; however, we use the term corner instead to describe the shape as seen from a cross-sectional view along the wire axis.

Two representative 2D slices of the Au 111 Bragg peaks are presented in Fig. 10a and 10b. The streaks originate from the nanowire side facets and, thus the angles between them illustrate the crystallographic orientation. Considering the fact that the amplitude of coherently scattered $\mathrm{X}$-ray is the Fourier transform of real space, the fringes define the size of the coherently illuminated crystal. Among the five Au NWs measured by BCD, two of them showed six streaks 
in their BCD pattern (Fig. 10a). This six-fold symmetry translates into a quasi-hexagonal shape with four $\{111\}$ and two $\{100\}$ facets as represented in the reconstructed cross-section presented in Fig. 10c. The BCD pattern of the other four NWs showed four streaks (Fig. 10b), which translates to a rhombic shape with all four facets determined as $\{111\}$ as seen in its reconstruction in Fig. 10d. The minimum area ratio between the $\{111\}$ and $\{100\}$ facets was 3.47, confirming $\{111\}$ as the crystallographically dominant facet in our Au NWs. By comparison, the corresponding ratio for a sharp Wulff shaped truncated rhombus is calculated to be 6.20 using $\gamma_{\{100\}} / \gamma_{\{111\}}$ [85]. The NWs presented in this work thus deviate from the Wulff construction by having rhombic or parallelogram shaped NWs, but tend to agree with the predictions assuming $\{111\}$ as the prevalent facet. With one set of the $\{111\}$ facets growing prevalently, nanoribbons can ultimately form as illustrated in Fig. 10f. These variances in shape are in agreement with other BCD results [84] and electron microscopy observations $[49,65,82]$.

In this section, we consider surface diffusional activity on our experimentally determined surface crystallography as the rate-limiting step for dislocation nucleation by comparing the surface self-diffusion barrier $\left(E_{\mathrm{d}}\right)$ and our experimentally obtained $\Delta G_{\text {act }}$. Although the activation energy for adatom jumps on $\{111\}$ facets were comparable to our activation Gibbs free energy for the $10 \mathrm{~nm}$ coated NWs at the most probable nucleation condition, further discussion is needed to consider adatom diffusion on a flat surface as the direct cause for dislocation nucleation. Indeed, there are multiple surface diffusion mechanisms including exchange mechanisms, and more combinations are possible with atomic steps on a given surface [86,87]. Indeed, the Ehrlich-Schwoebel (ES) barrier for $\mathrm{Au}\{111\}$ was reported to be the lowest among FCC crystals (Ni, Cu, Pd, Ag, Pt) [87] indicating that $\mathrm{Au}\{111\}$ facets tend to flatten well. In fact, $\mathrm{Au}\{111\}$ surfaces are reported to be stable enough to remain crystalline even up to $\mathrm{T}_{\mathrm{M}}[88]$. 
However, the corners of NWs are never atomically sharp, thus making atomic steps necessary to reconcile observations of rounded corners $[49,65]$. These atomic terraces can be a stress concentration site as well as one must consider diffusion from one layer to another.

Several atomistic simulation studies reported that the first partial dislocation nucleates at the most obtuse angle. For $\langle 110\rangle$ rhombic Au NWs in tension, atomistic simulations predict obtuse corners as the preferential nucleation site $[42,46]$. Atomistic simulations on $<110>$ Wulff shaped $\mathrm{Au}$ NWs report that the first leading partial dislocation nucleates at a corner shared by $\{111\}$ and $\{100\}$ [47]. Thus, we presume that rate-limiting diffusional activity enabling dislocation nucleation occurs at the smoothest transition corner, assuming the existence of $\{111\}$ vicinal surfaces with $\{100\}$ type ledges. For such a Au surface with $\{111\}$ terraces and $\{100\}$ ledges, an activation energy of $0.316 \mathrm{eV}$ was calculated by action-driven molecular dynamics (ADMD) for the parallel hopping along the ledge [87]. An exchange mechanism is preferred over hopping for the vertical movement across the step, which is predicted to be $0.694 \mathrm{eV}$ and $0.293 \mathrm{eV}$ for ascending and descending, respectively. Thus, hopping vertically up may not be a preferred mechanism, but rather the parallel hopping or an adatom descending across the step could trigger the dislocation nucleation event. In addition to surface steps, the step itself can contain kinks. Thus, rather than having straight terraces along the NW, the terraces themselves can have discontinuities. Interestingly, hopping onto a kink shows a large reduction of activation energy compared to barriers for straight steps. The activation energy depends on the original position of the atom relative to the kink, with a minimum value of $0.056 \mathrm{eV}$. Thus, among various surface diffusion mechanisms, the precursor event for dislocation nucleation involving either atom descending across a step or an atom attaching to a kink is most consistent with our results. The 
ALD coating can kinetically hinder such processes by dragging the adatom movements, thereby impeding the nucleation of a dislocation.

Finally, we describe the role of the ALD coating in the corner of the modified NW surface. Due to the difference in surface energies between $\{111\}$ and $\{100\}$, selective adsorption can occur in the initial cycles of ALD which can lead to non-conformal coatings. This phenomenon was observed in our study by measuring the thickness of the coating with TEM along different zone axes (Fig. 3d). We have measured a $3 \mathrm{~nm}$ coated nanowire with TEM and the average thickness of the coating on $\{100\}$ and $\{111\}$ facets were $5.3 \mathrm{~nm}$ and $3.5 \mathrm{~nm}$, respectively. The higher surface energy of $\{100\}$ over $\{111\}$ allows for more facile $\mathrm{Al}_{2} \mathrm{O}_{3}$ capture. Irregular coatings with insufficient layer thickness or even island formation can lead to stress concentrations, which may explain the lower $\Delta U_{\text {act }}$ and $\Omega$ of the $3 \mathrm{~nm}$ coated NWs compared to uncoated NWs. A non-monotonic trend in the activation parameters as a function of coating thickness (See Table 1) can be attributed to a non-conformal coating layer that arises from the physisorption process in the initial stages of the ALD process. In principle, Au atoms and the molecules used during the $\mathrm{ALD}$ process to form $\mathrm{Al}_{2} \mathrm{O}_{3}$ layers are unreactive, unlike with sulfur, where AuS is formed that mobilizes the gold surface atoms [89]. Thus, physisorbtion is the most probable means of adhesion between the two species by weak Van der Waals forces [90]. It has been reported that adhesion of $\mathrm{Au}\{111\}$ and $\{100\}$ on $\{0001\}$ sapphire substrates are similarly poor, and even poorer for polycrystalline alumina substrates owing to low heats of adhesion, suggesting the coating is physisorbed to Au NW surface $[91,92]$. Since physisorbtion forms weak interfaces, the schematic depiction of a self-terminating ALD process is likely not fully accurate. In reality, a scenario between Volmer-Weber and Stranski-Krastanov growth modes via formation of islands at low cycle numbers may occur [90,93]. During the first 40 ALD cycles to 
target $3 \mathrm{~nm}$ thick coatings, these islands can lead to a non-conformal coating layer, particularly on $\{111\}$ facets as illustrated in Fig. 11a. With such coating morphologies where the diffusional length is much smaller than the island spacing $(\lambda)$, the influence of the coating on surface diffusion on $\{111\}$ predominant facet may be insignificant. In contrast, after sufficient additional ALD cycles, the $10 \mathrm{~nm}$ coating can become conformal (Fig. 11b), and thus is able to effectively retard surface mobility.

\subsection{Nucleation site and attempt frequency}

Our attempt frequency from the exponential prefactors $\left(N \nu_{0}\right)$ with $N=1$ is 11 orders of magnitude lower than those associated with atomic vibrational frequencies $\left(\sim 10^{12} \mathrm{~s}^{-1}\right)$, and worthy of further discussion. Small numbers of equivalent nucleation sites $(N)$ are plausible within the weakest link framework, for example by having a highly susceptible nucleation site like a specific kink configuration among along a step leaving with an alternative explanation for the main attempt frequency as discussed in $[51,94]$.

Whereas the attempt frequency for surface dislocation nucleation has been assumed to be of the order of the Debye frequency in most MD simulations, experiments on Pd NWs in tension revealed that the attempt frequency can be much lower than the atomic vibration frequency, which led to the proposal of a collective attempt mechanism for surface nucleation [34]. In addition, recent in situ TEM experiments on nano-sized Ag demonstrated copious surface diffusion accommodating large amounts of plasticity during deformation, manifest as liquid-like pseudoelasticity in $\mathrm{Ag}$ nanoparticles and super-elongation in room temperature tension of $\mathrm{Ag}$ NW [37,38]. In particular, the latter work proposes facile healing of surface ledges created by dislocation slip via surface diffusion. While the authors claim that surface diffusion is provided 
assistance from the surface atomic step formed by the dislocation nucleation, we envisage this mechanism occurring at pre-existing surface steps near corners that act as preferred nucleation sites. Presumably, the existing surface steps can fluctuate along the NW by adatom diffusion

until the formation of a spatially extended nucleation site large enough to host a partial dislocation loop embryo, which can give much lower time scales for the attempt frequency. The emerging hypothesis is that the collective action of individual surface diffusion events serves as the rate-limiting step for the nucleation of a dislocation segment, which is reflected in the measurements of the nucleation strength.

\subsection{Tailoring mechanical response by control of the surface/interface}

By comparing the strength distributions between the uncoated and coated Au NWs, we have shown that adhesion of the coating on the surface mediates dislocation nucleation. In the report of pseudoelasticity in Ag nanoparticles, indentation loading leads to a relatively large diffusion zone [37] whereas tensile tests of Ag NW exhibit only surface diffusion [38]. Although the two studies have different sizes and loading modes, one may note that the zone participating in diffusion can lead to a completely different mechanical response. These recent findings as well as the studies on Pd NWs [34] and our Au NWs leads to the conclusion that in the sub-100 nm regime of metal nanostructures, the relative contribution of two deformation mechanisms can be tuned as demanded by the specific application.

Surface mobility is evidently critical for the dynamic healing process underpinning slipactivated surface diffusion and ultimately superplasticity as shown in Ag NWs in tension [38]. In the referenced study, surface diffusion of $\mathrm{Pt}$ (which has a higher $E_{\mathrm{d}}$ than $\mathrm{Ag}$ ) was not sufficiently rapid to accommodate stable plasticity and reside in a dislocation slip-dominated 
deformation mode. The study also proposed a critical limit of the diameter ( $70 \mathrm{~nm}$ for $\mathrm{Ag}$ and $\sim 10 \mathrm{~nm}$ for Pt) to maintain the uniform plasticity via diffusion aided healing process. The $E_{\mathrm{d}}$ of Au lies in between $\mathrm{Pt}$ and $\mathrm{Ag}[86,87]$, thus the cross over point for the Au can be reasonably estimated to fall between those two limits. Strong interfaces may make the core yield at even higher stress, yet potentially lead to stochastic failure due to statistically distributed flaws in addition to thermal fluctuations. On the other hand, relatively weak interfaces can dynamically heal the surface, thereby narrowing the dislocation nucleation stress distribution owing to larger activation volumes. Thus, the bonding at the interface should be optimized to sufficiently heighten the energy barrier of the rate-limiting step for dislocation nucleation while still utilizing in-plane interfacial diffusion to dynamically heal either the pre-existing surface flaws, or ledges formed as a result of previously nucleated dislocation. Taken as a whole, these strategies could raise strengths while simultaneously shifting to a more deterministic mechanical response without compromising ductility.

\section{Conclusion}

In this paper, we compare strength distributions of surface modified Au NWs measured in tension to demonstrate that the strength determined by dislocation nucleation from the surface can be tailored using ultrathin coatings. We extracted thermal activation parameters from the experimental strength data using a model developed from TST to conclude that tailoring surface diffusional activity is the key factor in controlling surface dislocation nucleation in these $\mathrm{Au}$ NWs. The results of over 40 NWs tested in tension were used to draw the following conclusions:

- The yield strength of PVD grown, defect-scarce <110> Au NWs in tension was measured to approach the theoretical strength (over $1 \mathrm{GPa}$ ), which is substantially higher than its 
bulk single-crystalline counterpart and is ascribed to nucleation of partial dislocation from the surface. The distinctive slip traces from the postmortem TEM micrographs indicate that partial dislocation events occur on $\{111\}$ planes, which agrees with previous simulations and measurements of $<110\rangle$ Au NWs. The strength distribution exhibits a weak size dependence in agreement with the characteristics of a thermally activated nucleation event as described by TST.

- The yield strengths of the Au cores in coated NWs remain high, and the nucleation mechanism underlying incipient plasticity is consistent with that of the uncoated NWs. While the thinner coating causes subtle increases in the scatter of the strength distribution, the thicker coatings show a pronounced narrowing of the distribution with a shift toward high strength values. Sufficient coverage of the coating can kinetically hinder surface mobility as evidenced by increases in both the activation energy and the activation volume for dislocation nucleation.

- The activation Gibbs free energy implies that surface diffusional activity is the ratelimiting step for the nucleation event at finite temperature. An athermal strength of 2.26 GPa was derived from temperature controlled tensile tests for $10 \mathrm{~nm}$ coated Au NWs, suggesting that the coating has minimal effect on the $0 \mathrm{~K}$ strength.

- Tailoring the interfacial shear strength force between the core and the coating may be the key optimization parameter for tailoring the ductility and strength in the surface dislocation nucleation mediated regime.

Our work outlines a strategy for tailoring dislocation nucleation-controlled strength in surface or interface-dominated nanostructures at the extreme upper limit of strength. This 
approach appears to mitigate some of the primary obstacles facing the deployment of nanostructures that lead to unreliable mechanical stability, including anelastic behavior or stochastic failure.

\section{Acknowledgements}

This research was primarily supported by the National Science Foundation through a CAREER Award \#DMR-1056293. The authors acknowledge support of the A*MIDEX grant (ANR-11-IDEX-0001-02) funded by the French Government «Investissements d'Avenir» program. DSG and OT acknowledge partial support from the NSF MRSEC Program through DMR 1720256 (IRG-1). The research reported here made use of shared facilities of the UCSB MRSEC (NSF DMR 1720256), a member of the Materials Research Facilities Network (www.mrfn.org). The authors also thank the support of the staff and facilities at the Penn Nanoscale Characterization Facility at the University of Pennsylvania and TEM assistance from Aidan Taylor at UCSB. 


\section{References}

[1] C. Li, L. Wang, H. Liu, J. Wang, Z. Liao, D. Yu, Giant negative magnetoresistance induced by the chiral anomaly in individual Cd3As2 nanowires, Nat. Commun. 6 (2015) 1-7. doi:10.1038/ncomms10137.

[2] W.F. Paxton, K.C. Kistler, C.C. Olmeda, A. Sen, S.K.S. Angelo, Y. Cao, T.E. Mallouk, P.E. Lammert, V.H. Crespi, Catalytic Nanomotors : Autonomous Movement of Striped Nanorods, J. Am. Chem. Soc. 126 (2004) 13424-13431. doi:10.1021/ja047697z.

[3] Y. Kondo, K. Takayanagi, Synthesis and Characterization of Helical Multi-Shell Gold Nanowires, Science (80-. ). 289 (2000) 606-609.

[4] Y. Wu, J. Xiang, C. Yang, W. Lu, C.M. Lieber, Single-crystal metallic nanowires and metal / semiconductor nanowire heterostructures, Nature. 430 (2004) 61-65.

[5] F. Guo, H. Azimi, Y. Hou, T. Przybilla, M. Hu, C. Bronnbauer, S. Langner, E. Spiecker, K. Forberich, C.J. Brabec, High-performance semitransparent perovskite solar cells with solution-processed silver nanowires as top electrodes, Nanoscale. 7 (2015) 1642-1649. doi:10.1039/c4nr06033d.

[6] J. Lee, S.T. Connor, Y. Cui, P. Peumans, E. Engineering, S.U. V, Solution-Processed Metal Nanowire Mesh Transparent Electrodes, Nano Lett. 8 (2008) 689-692. doi:10.1021/n1073296g.

[7] L. Cao, P. Fan, A.P. Vasudev, J.S. White, Z. Yu, W. Cai, J.A. Schuller, S. Fan, M.L. Brongersma, Semiconductor Nanowire Optical Antenna Solar Absorbers, Nano Lett. 10 (2010) 439-445. doi:10.1021/n19036627. 
[8] M.D. Uchic, D.M. Dimiduk, J.N. Florando, W.D. Nix, Sample dimensions influence strength and crystal plasticity, Science (80-. ). 305 (2004) 986-989. doi:10.1126/science.1098993.

[9] J.R. Greer, W.C. Oliver, W.D. Nix, Size dependence of mechanical properties of gold at the micron scale in the absence of strain gradients, Acta Mater. 53 (2005) 1821-1830. doi:10.1016/j.actamat.2004.12.031.

[10] H. Bei, S. Shim, E. George, M. Miller, E. Herbert, G. Pharr, Compressive strengths of molybdenum alloy micro-pillars prepared using a new technique, Scr. Mater. 57 (2007) 397-400. doi:10.1016/j.scriptamat.2007.05.010.

[11] B. Wu, A. Heidelberg, J.J. Boland, Mechanical properties of ultrahigh-strength gold nanowires., Nat. Mater. 4 (2005) 525-9. doi:10.1038/nmat1403.

[12] S. Hoffmann, I. Utke, B. Moser, J. Michler, S.H. Christiansen, V. Schmidt, S. Senz, P. Werner, Measurement of the Bending Strength of Vapor - Liquid - Solid Grown Silicon Nanowires, (2006) 1-4. doi:10.1021/n1052223z.

[13] B. Wu, A. Heidelberg, J.J. Boland, J.E. Sader, Microstructure-Hardened Silver, (2006) 15. doi:10.1021/n1052427f.

[14] S.S. Brenner, Tensile Strength of Whiskers, J. Appl. Phys. 27 (1956) 1484-1491. doi:10.1063/1.1722294.

[15] G. Richter, K. Hillerich, D.S. Gianola, R. Mönig, O. Kraft, C.A. Volkert, Ultrahigh strength single crystalline nanowhiskers grown by physical vapor deposition, Nano Lett. 9 (2009) 3048-3052. doi:10.1021/n19015107. 
[16] H.S. Park, Surface stress effects on the resonant properties of silicon nanowires, J. Appl. Phys. 103 (2008) 123504. doi:10.1063/1.2939576.

[17] G. Jing, H. Duan, X. Sun, Z. Zhang, J. Xu, Y. Li, J. Wang, D. Yu, Surface effects on elastic properties of silver nanowires: Contact atomic-force microscopy, Phys. Rev. B. 73 (2006) 1-6. doi:10.1103/PhysRevB.73.235409.

[18] J. Diao, K. Gall, M.L. Dunn, Surface-stress-induced phase transformation in metal nanowires., Nat. Mater. 2 (2003) 656-660. doi:10.1038/nmat977.

[19] J. Diao, K. Gall, M.L. Dunn, Surface stress driven reorientation of gold nanowires, Phys. Rev. B - Condens. Matter Mater. Phys. 70 (2004) 1-9. doi:10.1103/PhysRevB.70.075413.

[20] R.E. Miller, V.B. Shenoy, Size-dependent elastic properties of nanosized structural elements, Nanotechnology. 11 (2000) 139-147. doi:10.1088/0957-4484/11/3/301.

[21] H. Mecking, U.F. Kocks, Kinetics of flow and strain-hardening, Acta Metall. 29 (1981) $1865-1875$.

[22] R. Labusch, A Statistical Theory of Solid Solution Hardening, Phys. Status Solidi. 41 (1970) 659-669.

[23] A.J. Ardell, Precipitation Hardening, Metall. Trans. A. 16A (1985) 2131-2165.

[24] D.M. Dimiduk, M.D. Uchic, T.A. Parthasarathy, Size-affected single-slip behavior of pure nickel microcrystals, Acta Mater. 53 (2005) 4065-4077. doi:10.1016/j.actamat.2005.05.023.

[25] O. Kraft, P.A. Gruber, R. Mönig, D. Weygand, Plasticity in Confined Dimensions, Annu. 
Rev. Mater. Res. 40 (2010) 293-317. doi:10.1146/annurev-matsci-082908-145409.

[26] S.H. Oh, M. Legros, D. Kiener, G. Dehm, In situ observation of dislocation nucleation and escape in a submicrometre aluminium single crystal, Nat. Mater. 8 (2009) 95-100. doi:Doi 10.1038/Nmat2370.

[27] Y. Gao, H. Bei, Strength statistics of single crystals and metallic glasses under small stressed volumes, Prog. Mater. Sci. 82 (2016) 118-150. doi:http://dx.doi.org/10.1016/j.pmatsci.2016.05.002.

[28] J.R. Greer, J.T.M. De Hosson, Plasticity in small-sized metallic systems: Intrinsic versus extrinsic size effect, Prog. Mater. Sci. 56 (2011) 654-724. doi:10.1016/j.pmatsci.2011.01.005.

[29] H. Bei, S. Shim, G.M. Pharr, E.P. George, Effects of pre-strain on the compressive stressstrain response of Mo-alloy single-crystal micropillars, Acta Mater. 56 (2008) 4762-4770. doi:10.1016/j.actamat.2008.05.030.

[30] C.R. Weinberger, W. Cai, Plasticity of metal nanowires, J. Mater. Chem. 22 (2012) 3277. doi: $10.1039 / \mathrm{c} 2 \mathrm{jm} 13682 \mathrm{a}$.

[31] T. Zhu, J. Li, A. Samanta, A. Leach, K. Gall, Temperature and Strain-Rate Dependence of Surface Dislocation Nucleation, Phys. Rev. Lett. 100 (2008) 025502. doi:10.1103/PhysRevLett.100.025502.

[32] S. Brochard, P. Hirel, L. Pizzagalli, J. Godet, Elastic limit for surface step dislocation nucleation in face-centered cubic metals: Temperature and step height dependence, Acta Mater. 58 (2010) 4182-4190. doi:10.1016/j.actamat.2010.04.009. 
[33] D.H. Warner, W. a. Curtin, Origins and implications of temperature-dependent activation energy barriers for dislocation nucleation in face-centered cubic metals, Acta Mater. 57 (2009) 4267-4277. doi:10.1016/j.actamat.2009.05.024.

[34] L.Y. Chen, M.-R. He, J. Shin, G. Richter, D.S. Gianola, Measuring surface dislocation nucleation in defect-scarce nanostructures., Nat. Mater. 14 (2015) 707-713. doi:10.1038/nmat4288.

[35] L.Y. Chen, G. Richter, J.P. Sullivan, D.S. Gianola, Lattice anharmonicity in defect-free Pd nanowhiskers, Phys. Rev. Lett. 109 (2012) 1-5. doi:10.1103/PhysRevLett.109.125503.

[36] G. Cheng, C. Miao, Q. Qin, J. Li, F. Xu, H. Haftbaradaran, E.C. Dickey, H. Gao, Y. Zhu, Large anelasticity and associated energy dissipation in single-crystalline nanowires, Nat Nanotechnol. 10 (2015) 687-691. doi:10.1038/nnano.2015.135.

[37] J. Sun, L. He, Y.-C. Lo, T. Xu, H. Bi, L. Sun, Z. Zhang, S.X. Mao, J. Li, Liquid-like pseudoelasticity of sub-10-nm crystalline silver particles., Nat. Mater. 13 (2014). doi:10.1038/nmat4105.

[38] L. Zhong, F. Sansoz, Y. He, C. Wang, Z. Zhang, S.X. Mao, Slip-activated surface creep with room-temperature super-elongation in metallic nanocrystals, Nat. Mater. 1 (2016) 18. doi:10.1038/nmat4813.

[39] N. Mameka, On the impact of capillarity for strength at the nanoscale, Nat. Commun. (n.d.) 1-8. doi:10.1038/s41467-017-01434-2.

[40] J.J. Métois, A. Saúl, P. Müller, Measuring the surface stress polar dependence, Nat. Mater. 4 (2005) 238-242. doi:10.1038/nmat1328. 
[41] Q.J. Li, B. Xu, S. Hara, J. Li, E. Ma, Sample-size-dependent surface dislocation nucleation in nanoscale crystals, Acta Mater. 145 (2018) 19-29. doi:10.1016/j.actamat.2017.11.048.

[42] A.T. Jennings, C.R. Weinberger, S.-W. Lee, Z.H. Aitken, L. Meza, J.R. Greer, Modeling dislocation nucleation strengths in pristine metallic nanowires under experimental conditions, Acta Mater. 61 (2013) 2244-2259. doi:10.1016/j.actamat.2012.12.044.

[43] J. Diao, K. Gall, M.L. Dunn, Yield Strength Asymmetry in Metal Nanowires, Nano Lett. 4 (2004) 1863-1867. doi:10.1021/n10489992.

[44] S. Aubry, K. Kang, S. Ryu, W. Cai, Energy barrier for homogeneous dislocation nucleation: Comparing atomistic and continuum models, Scr. Mater. 64 (2011) 10431046. doi:10.1016/j.scriptamat.2011.02.023.

[45] S. Ryu, K. Kang, W. Cai, Predicting the dislocation nucleation rate as a function of temperature and stress, J. Mater. Res. 26 (2011) 2335-2354. doi:10.1557/jmr.2011.275.

[46] C.R. Weinberger, A.T. Jennings, K. Kang, J.R. Greer, Atomistic simulations and continuum modeling of dislocation nucleation and strength in gold nanowires, J. Mech. Phys. Solids. 60 (2012) 84-103. doi:10.1016/j.jmps.2011.09.010.

[47] A. Sedlmayr, E. Bitzek, D.S. Gianola, G. Richter, R. Mönig, O. Kraft, Existence of two twinning-mediated plastic deformation modes in Au nanowhiskers, Acta Mater. 60 (2012) 3985-3993. doi:10.1016/j.actamat.2012.03.018.

[48] S. Ogata, J. Li, S. Yip, Energy landscape of deformation twinning in bcc and fcc metals, (2005) 1-11. doi:10.1103/PhysRevB.71.224102. 
[49] B. Roos, B. Kapelle, G. Richter, C.A. Volkert, Surface dislocation nucleation controlled deformation of Au nanowires, Appl. Phys. Lett. 105 (2014) 201908. doi:10.1063/1.4902313.

[50] D. Wu, T.G. Nieh, Incipient plasticity and dislocation nucleation in body-centered cubic chromium, Mater. Sci. Eng. A. 609 (2014) 110-115. doi:10.1016/j.msea.2014.04.107.

[51] J. Mason, a. Lund, C. Schuh, Determining the activation energy and volume for the onset of plasticity during nanoindentation, Phys. Rev. B. 73 (2006) 054102. doi:10.1103/PhysRevB.73.054102.

[52] S. Ryu, K. Kang, W. Cai, Entropic effect on the rate of dislocation nucleation., Proc. Natl. Acad. Sci. U. S. A. 108 (2011) 5174-5178. doi:10.1073/pnas.1017171108.

[53] D. Chachamovitz, D. Mordehai, The Stress-Dependent Activation Parameters for Dislocation Nucleation in Molybdenum Nanoparticles, Sci. Rep. 8 (2018) 1-10. doi:10.1038/s41598-018-21868-y.

[54] J. Li, K.J. Van Vliet, T. Zhu, S. Yip, S. Suresh, Atomistic mechanisms governing elastic limit and incipient plasticity in crystals., Nature. 418 (2002) 307-10. doi:10.1038/nature00865.

[55] A.T. Jennings, C.R. Weinberger, S.-W. Lee, Z.H. Aitken, L. Meza, J.R. Greer, Modeling dislocation nucleation strengths in pristine metallic nanowires under experimental conditions, Acta Mater. 61 (2013) 2244-2259. doi:10.1016/j.actamat.2012.12.044.

[56] T. Zhu, J. Li, Ultra-strength materials, Prog. Mater. Sci. 55 (2010) 710-757. doi:10.1016/j.pmatsci.2010.04.001. 
[57] S. Jakschik, U. Schroeder, T. Hecht, M. Gutsche, H. Seidl, J.W. Bartha, Crystallization behavior of thin ALD-Al2O3 films, Thin Solid Films. 425 (2003) 216-220. doi:10.1016/S0040-6090(02)01262-2.

[58] Q. Yong, L. Seung-Mo, P. Anlian, U. Gosele, M. Knez, Rayleigh-instability-induced metal nanoparticle chains encapsulated in nanotubes produced by atomic layer deposition, Nano Lett. 8 (2008) 114-118. doi:10.1021/n10721766.

[59] D.S. Gianola, A. Sedlmayr, R. Mnig, C.A. Volkert, R.C. Major, E. Cyrankowski, S.A.S. Asif, O.L. Warren, O. Kraft, In situ nanomechanical testing in focused ion beam and scanning electron microscopes, Rev. Sci. Instrum. 82 (2011). doi:10.1063/1.3595423.

[60] D.J. Magagnosc, G. Kumar, J. Schroers, P. Felfer, J.M. Cairney, D.S. Gianola, Effect of ion irradiation on tensile ductility, strength and fictive temperature in metallic glass nanowires, Acta Mater. 74 (2014) 165-182. doi:10.1016/j.actamat.2014.04.002.

[61] K.F. Murphy, L.Y. Chen, D.S. Gianola, Effect of organometallic clamp properties on the apparent diversity of tensile response of nanowires., Nanotechnology. 24 (2013) 235704. doi:10.1088/0957-4484/24/23/235704.

[62] L.Y. Chen, S. Terrab, K.F. Murphy, J.P. Sullivan, X. Cheng, D.S. Gianola, Temperature controlled tensile testing of individual nanowires, Rev. Sci. Instrum. 85 (2014) 013901. doi:10.1063/1.4858815.

[63] J. Shin, T.W. Cornelius, S. Labat, F. Lauraux, M. Richard, G. Richter, In-situ Bragg coherent X-ray diffraction during tensile testing of an individual Au nanowire, J. Appl. Cryst. 1133 (2018) 35408. doi:10.1107/S1600576718004910. 
[64] J.R. Fienup, Phase retrieval algorithms: a comparison, Appl. Opt. 21 (1982) 2758. doi:10.1364/AO.21.002758.

[65] A. Sedlmayr, Experimental Investigations of Deformation Pathways in Nanowires, KIT Universität des Landes Baden-Württemberg und nationales Forschungszentrum in der Helmholtz-Gemeinschaft Diese, 2012.

[66] J.-H. Seo, Y. Yoo, N.-Y. Park, S.-W. Yoon, H. Lee, S. Han, S.-W. Lee, T.-Y. Seong, S.-C. Lee, K.-B. Lee, P.-R. Cha, H.S. Park, B. Kim, J.-P. Ahn, Superplastic deformation of defect-free Au nanowires via coherent twin propagation., Nano Lett. 11 (2011) 3499-502. doi:10.1021/n12022306.

[67] J. Diao, K. Gall, M.L. Dunn, Atomistic simulation of the structure and elastic properties of gold nanowires, J. Mech. Phys. Solids. 52 (2004) 1935-1962. doi:10.1016/j.jmps.2004.03.009.

[68] A. Cao, E. Ma, Sample shape and temperature strongly influence the yield strength of metallic nanopillars, Acta Mater. 56 (2008) 4816-4828. doi:10.1016/j.actamat.2008.05.044.

[69] M.K. Tripp, C. Stampfer, D.C. Miller, T. Helbling, C.F. Herrmann, C. Hierold, K. Gall, S.M. George, V.M. Bright, The mechanical properties of atomic layer deposited alumina for use in micro- and nano-electromechanical systems, Sensors Actuators A Phys. 130131 (2006) 419-429. doi:10.1016/j.sna.2006.01.029.

[70] S. Jen, J.A. Bertrand, S.M. George, Critical tensile and compressive strains for cracking of Al2O3 films grown by atomic layer deposition, J. Appl. Phys. 109 (2011) 1-11. 
doi:10.1063/1.3567912.

[71] D.C. Miller, R.R. Foster, S.-H. Jen, J.A. Bertrand, S.J. Cunningham, A.S. Morris, Y.-C. Lee, S.M. George, M.L. Dunn, Thermo-mechanical properties of alumina films created using the atomic layer deposition technique, Sensors Atuators A. 164 (2010) 58-67.

[72] K. Tapily, J.E. Jakes, D.S. Stone, P. Shrestha, D. Gu, H. Baumgart, A.A. Elmustafa, Nanoindentation Investigation of $\mathrm{HfO} 2$ and $\mathrm{Al} 2 \mathrm{O} 3$ Films Grown by Atomic Layer Deposition, J. Electrochem. Soc. 155 (2008) 545-551. doi:10.1149/1.2919106.

[73] B. Ilic, S. Krylov, H.G. Craighead, B. Ilic, S. Krylov, H.G. Craighead, Young's modulus and density measurements of thin atomic layer deposited films using resonant nanomechanics Young's modulus and density measurements of thin atomic layer deposited films using resonant nanomechanics, J. Appl. Phys. 108 (2010) 1-11. doi:10.1063/1.3474987.

[74] M.K. Tripp, C. Stampfer, D.C. Miller, T. Helbling, C.F. Herrmann, C. Hierold, K. Gall, S.M. George, V.M. Bright, The mechanical properties of atomic layer deposited alumina for use in micro- and nano-electromechanical systems, Sensors Actuators, A Phys. 130131 (2006) 419-429. doi:10.1016/j.sna.2006.01.029.

[75] S.J. Bull, Mechanical response of atomic layer deposition alumina coatings on stiff and compliant substrates Mechanical response of atomic layer deposition alumina coatings on stiff and compliant substrates, J. Vac. Sci. Technol. A. 30 (2012) 1-8. doi:10.1116/1.3670401.

[76] O.M.E. Ylivaara, X. Liu, L. Kilpi, J. Lyytinen, D. Schneider, M. Laitinen, J. Julin, S. Ali, 
S. Sintonen, M. Berdova, E. Haimi, T. Sajavaara, H. Ronkainen, H. Lipsanen, J. Koskinen, S. Hannula, R.L. Puurunen, Aluminum oxide from trimethylaluminum and water by atomic layer deposition: The temperature dependence of residual stress, elastic modulus, hardness and adhesion Ł̧, Thin Solid Films. 552 (2014) 124-135. doi:10.1016/j.tsf.2013.11.112.

[77] A.H.W. Ngan, L. Zuo, P.C. Wo, Size dependence and stochastic nature of yield strength of micron-sized crystals: a case study on Ni3Al, Proc. R. Soc. A Math. Phys. Eng. Sci. 462 (2006) 1661-1681. doi:10.1098/rspa.2005.1645.

[78] C.L. Liu, J.M. Cohen, J.B. Adams, A.F. Voter, EAM study of surface self-diffusion of single adatoms of fcc metals Ni, Cu, Al, Ag, Au, Pd, and Pt, Surf. Sci. 253 (1991) 334344.

[79] S.M. Foiles, M.I. Baskes, M.S. Daw, Embedded-atom-method functions for the fcc metals Cu, Ag, Au, Ni, Pd, Pt, and their alloys, Phys. Rev. B. 33 (1986) 7983-7991. doi:10.1103/PhysRevB.33.7983.

[80] S. V Eremeev, A.G. Lipnitskii, A.I. Potekaev, E. V Chulkov, Activation energy for diffusion of point defects at the surfaces of F.C.C. metals, Russ. Phys. J. 40 (1997) 584589. doi:10.1007/BF02766393.

[81] Y. Hiki, A.V. Granato, Anharmonicity in Noble Metals; Higher Order Elastic Constants, Phys. Rev. 144 (1966) 411-419. doi:10.1017/CBO9781107415324.004.

[82] L.Y. Chen, Deformation Mechanisms in Pd Nanowhiskers Deformation Mechanisms in Pd Nanowhiskers, Publicly Access. Penn Diss. Paper 1049 (2014). 
[83] S. Labat, M.I. Richard, M. Dupraz, M. Gailhanou, G. Beutier, M. Verdier, F. Mastropietro, T.W. Cornelius, T.U. Schülli, J. Eymery, O. Thomas, Inversion Domain Boundaries in GaN Wires Revealed by Coherent Bragg Imaging, ACS Nano. 9 (2015) 9210-9216. doi:10.1021/acsnano.5b03857.

[84] C. Leclere, T.W. Cornelius, Z. Ren, A. Davydok, J.-S. Micha, O. Robach, G. Richter, L. Belliard, O. Thomas, In situ bending of an Au nanowire monitored by micro Laue diffraction, J. Appl. Crystallogr. 48 (2015) 291-296. doi:10.1107/S1600576715001107.

[85] A.M. Rodriguez, G. Bozzolo, J. Ferrante, Multilayer relaxation and surface energies of fcc and bcc metals using equivalent crystal theory, Surf. Sci. 289 (1993) 100-126.

[86] S.Y. Kim, I. Lee, S. Jun, Transition-pathway models of atomic diffusion on fcc metal surfaces. I. Flat surfaces, Phys. Rev. B. 76 (2007) 1-15. doi:10.1103/PhysRevB.76.245407.

[87] S.Y. Kim, I. Lee, S. Jun, Transition-pathway models of atomic diffusion on fcc metal surfaces . II . Stepped surfaces, Phys. Rev. B. 76 (2007) 1-20. doi:10.1103/PhysRevB.76.245408.

[88] F. Ercolessi, W. Andreoni, E. Tosatti, Melting of small gold particles: Mechanism and size effects, Phys. Rev. Lett. 66 (1991) 911-914. doi:10.1103/PhysRevLett.66.911.

[89] M.M. Biener, J. Biener, C.M. Friend, Sulfur-induced mobilization of Au surface atoms on Au(1 11 1) studied by real-time STM, Surf. Sci. 601 (2007) 1659-1667. doi:10.1016/j.susc.2007.01.041.

[90] R.L. Puurunen, Surface chemistry of atomic layer deposition: A case study for the 
trimethylaluminum/water process, J. Appl. Phys. 97 (2005). doi:10.1063/1.1940727.

[91] H. Sadan, W.D. Kaplan, Au-Sapphire (0001) solid-solid interfacial energy, J. Mater. Sci. 41 (2006) 5099-5107. doi:10.1007/s10853-006-0437-5.

[92] R. Gomer, Diffusion of adsorbates on metal surfaces, Reports Prog. Phys. 53 (1990) 9171002. doi:10.1088/0034-4885/53/7/002.

[93] S.M. George, Atomic Layer Deposition : An Overview, (2010) 111-131.

[94] D. Perez, B.P. Uberuaga, A.F. Voter, The parallel replica dynamics method - Coming of age, Comput. Mater. Sci. 100 (2015) 90-103. doi:10.1016/j.commatsci.2014.12.011. 
Table 1. Activation parameters obtained from room temperature fitting of the room temperature yield strength distributions

\begin{tabular}{lllll}
\hline & Mean Yield Strength $(\mathbf{G P a})$ & $\boldsymbol{\Delta} \boldsymbol{U}_{\text {act }}(\mathbf{e V})$ & $\mathbf{\Omega}\left(\mathbf{b}_{\mathbf{p}}^{\mathbf{3}}\right)$ & $\boldsymbol{N \boldsymbol { v } _ { \mathbf { 0 } } ( \mathbf { s } ^ { \mathbf { - 1 } } )}$ \\
\hline Uncoated & $1.098 \pm 0.391$ & 0.252 & 3.69 & 33.83 \\
$\mathbf{3} \mathbf{~ n m ~ C o a t e d ~}$ & $1.297 \pm 0.484$ & 0.242 & 2.07 & 49.30 \\
$\mathbf{1 0} \mathbf{~ n m ~ C o a t e d ~}$ & $1.496 \pm 0.305$ & 0.332 & 6.01 & 18.63 \\
\hline
\end{tabular}




\section{Figure caption}

Figure 1. a) BF TEM image of uncoated Au NW b) HR TEM image of uncoated Au NW c) BF TEM image of Au NW coated with $\mathrm{ALD}^{\mathrm{Al}_{2} \mathrm{O}_{3}}$ d) HR TEM image of coated $\mathrm{Au}$ NW e) Schematic of coated NW with a Au NW diameter $d_{\text {core }}$ and coating thickness $t$ f) Au core diameter statistics g) Coating thickness statistics for $3 \mathrm{~nm}$ (40 cycle ALD) and $10 \mathrm{~nm}$ (200 cycle ALD) target thickness.

Figure 2. a) Representative engineering tensile stress-strain curves for each type of NW: uncoated (including data from [65]) and coated with two different thicknesses. The load-bearing of the coating was subtracted for the $10 \mathrm{~nm}$ coated NWs. In cases where Pt-EBID markers could be deposited on the NWs and imaged during tensile testing, digital image correlation was employed to measure local strain. In other cases, the relative grip displacements were used to estimate nominal strain, which could incorporate the effects of grip compliance; thus, the strain values are nominal ones. Representative SEM images of fractured NWs for b) uncoated, c) $3 \mathrm{~nm}$ coated, and d) $10 \mathrm{~nm}$ coated Au NWs. Insets show higher magnification of fractured ends.

Figure 3. BF TEM images of plastically deformed NWs in all three sample configurations showing observations of stacking faults from a) an uncoated Au NW b) an Au NW with $3 \mathrm{~nm}$ thick $\mathrm{Al}_{2} \mathrm{O}_{3}$ coating, and c) an $\mathrm{Au} \mathrm{NW}$ with $10 \mathrm{~nm} \mathrm{Al}_{2} \mathrm{O}_{3}$ coating. The scale bar is common for all images. 
Figure 4. Characterization of slip bands in $10 \mathrm{~nm}$ coated NWs after tensile deformation, viewed from the a) $\langle 100\rangle$ b) $\langle 111\rangle$ and c) $\langle 110\rangle$ zone axes. d) Schematics of example slip traces as viewed from each zone axis. An identical NW is shown for a) and b).

Figure 5. BF TEM images of fractured NW segments for a) an uncoated Au NW, b) a $3 \mathrm{~nm}$ coated Au NW without twinned grains c) a $10 \mathrm{~nm}$ coated Au NW. Insets show the diffraction patterns. d) DF TEM image obtained from the circled diffraction spot in the inset of c), showing crystallographic reorientation of fracture tip.

Figure 6. a) Au NW affixed with Pt-EBID on a stage for tensile testing. Multiple Pt-EBID spot markers are placed on the stage and on the NW (marked by blue triangles) along the gauge length for local strain measurements. b) Engineering stress-strain curves for the wire in a) using two different strain measures. The wire strain corresponds to the local strain measured using DIC of the four markers on the wire gauge in a), and the grip strain is measured from the grip markers on the tensile stage. A fit to the unloading curve using the wire strain gives the Young's modulus of the composite core-shell structure, $E_{\text {eff. }}$ Two fits to the unloading curves are shown: one (green) to the initial portion of the unloading curve (giving $E_{\text {coat }}=102 \mathrm{GPa}$ with an $R^{2}$ value of 0.73 ) and another (red) to a larger portion of the unloading curve (giving $E_{\text {coat }}=122 \mathrm{GPa}$ with an $R^{2}$ value of 0.96$)$

Figure 7. Tensile yield strength as a function of Au NW diameter. The yield strength values for the uncoated Au NWs include data from both the current study and [65]. The values for the 10 
$\mathrm{nm}$ coated NWs were corrected for the Au core to exclude load bearing of the coating. The mean values and standard deviation for each group are represented in a box plot on the right side. In the case of the $10 \mathrm{~nm}$ coated NWs, we show values for two different $E_{\text {coat }}$ as labeled on the figure and corresponding to the two fits to the unloading curves shown in Figure 6. Given the higher quality of the fit for $E_{\text {coat }}=122 \mathrm{GPa}$, we employ these values of the $\mathrm{Au}$ core strength for all subsequent analyses.

Figure 8. Cumulative probability showing the room temperature yield strength distribution for NWs tested in tension in all three configurations. Overlaid is the fit (dotted lines) from Eq. 5 derived from TST to the experimentally measured yield strengths (symbols).

Figure 9. a) Stress-strain curves for $10 \mathrm{~nm}$ coated Au NWs tested at different temperatures. b) Measured yield (nucleation) stress overlaid on the probability density function model predictions. Probability values $P(\sigma, T)$ are normalized with respect to the maximum probability $P_{\max }(T)$ at each temperature; thus, each vertical slice represents the probability distribution at a given temperature, which collapses to a deterministic value in the athermal limit $(0 \mathrm{~K})$. The dashed contour lines represent the 5th (lower) and 95th (upper) percentile nucleation stresses.

Figure 10. Representative 2D slices of the Au 111 Bragg peaks obtained from BCD two for different NWs, demonstrating a) 6 streaks and b) 4 streaks in the pattern. c) - d) Reconstructed electron density maps of the NWs with Bragg peak patterns of a) and b), respectively. e) 
Reconstructed shape of a typical rhombic NW. All the figures share the same orientation. f) Schematic of possible cross-sectional shape of a NW derived from the truncated rhombic geometry of $<110>$ crystals.

Fig. 11. Drawing of proposed surface configurations for the a) $3 \mathrm{~nm}$ coating (40 cycle ALD) where islands of the ALD coating lead to incomplete coverage on $\{111\}$ facets, separated by a distance $\lambda$ that is larger than the expected diffusion length and b) $10 \mathrm{~nm}$ coating (200 cycle ALD) which exhibit more conformal coating layer. The surface affected zone (SAZ) is the near-surface region of the Au core which is influenced by surface diffusional activity, and ultimately where surface dislocation nucleation ensues. 

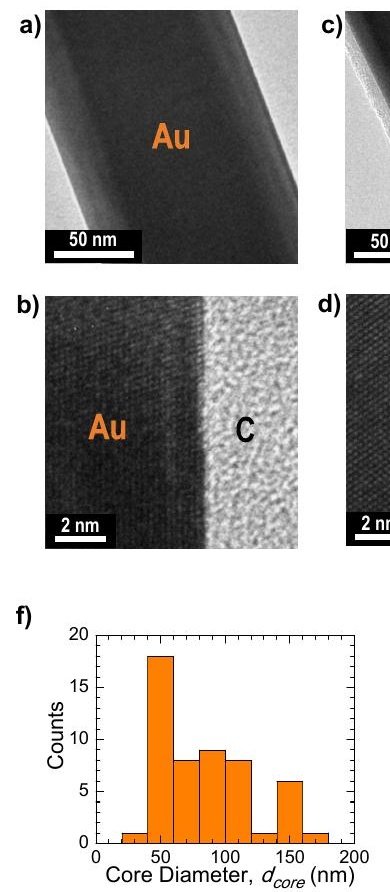

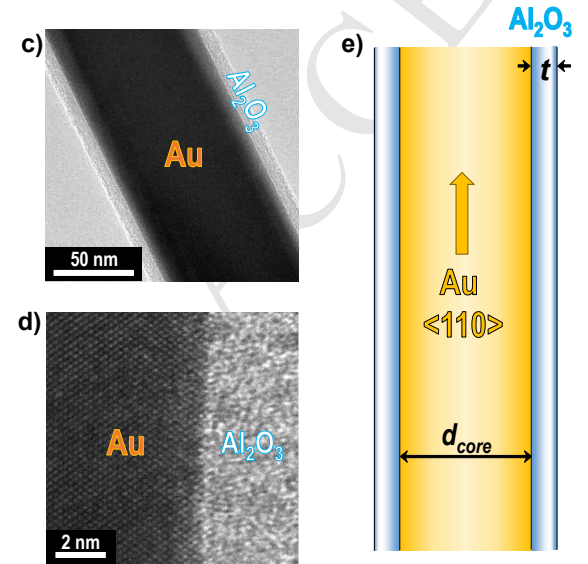

g)

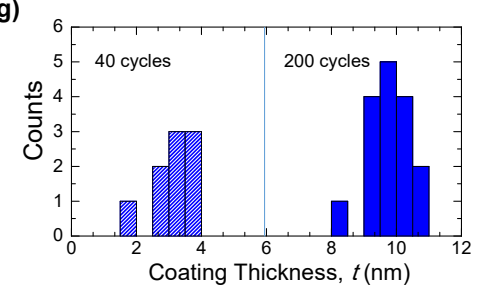


a)

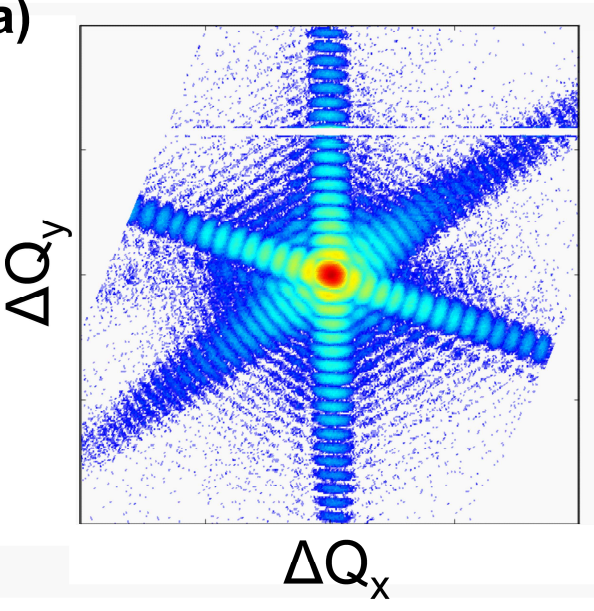

c)

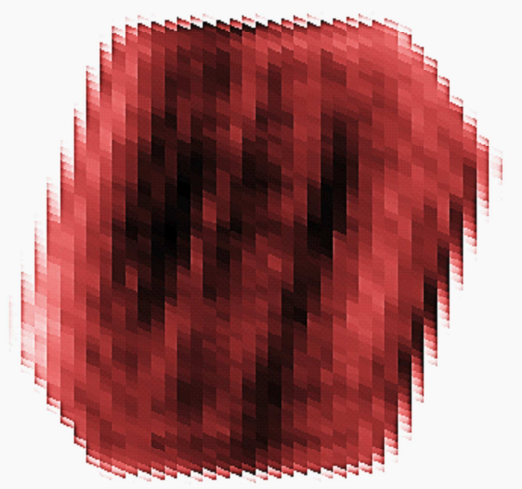

e)

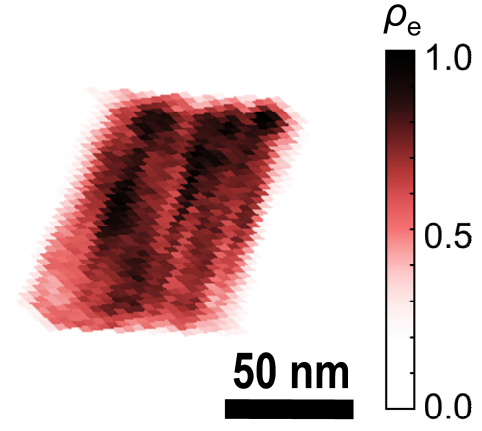

b)

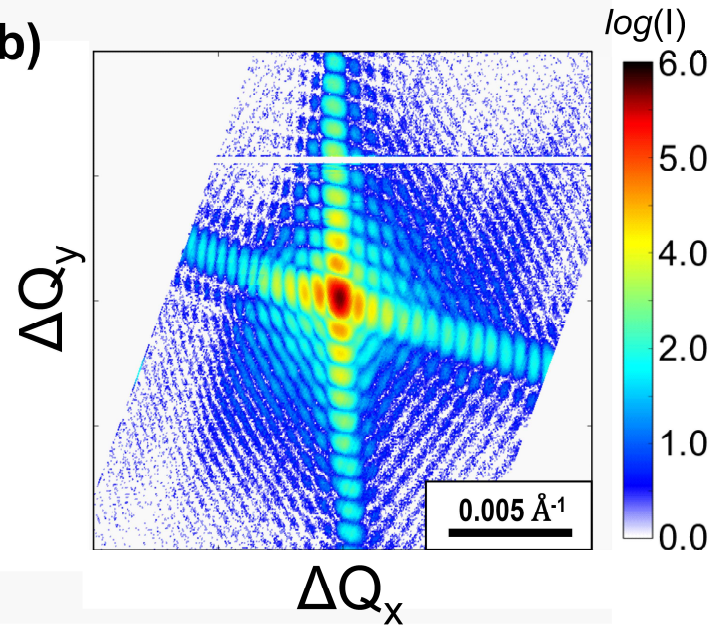

d)

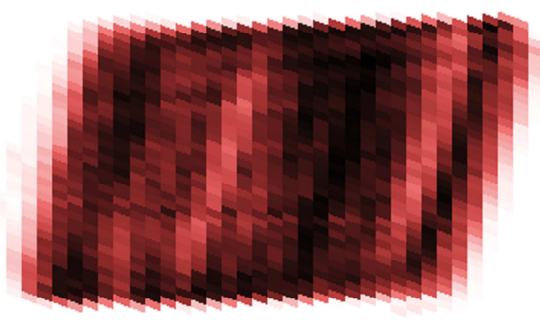

f)

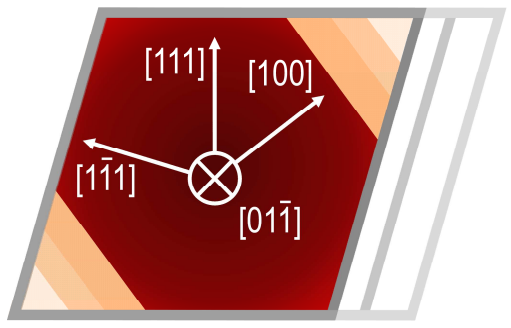


a)

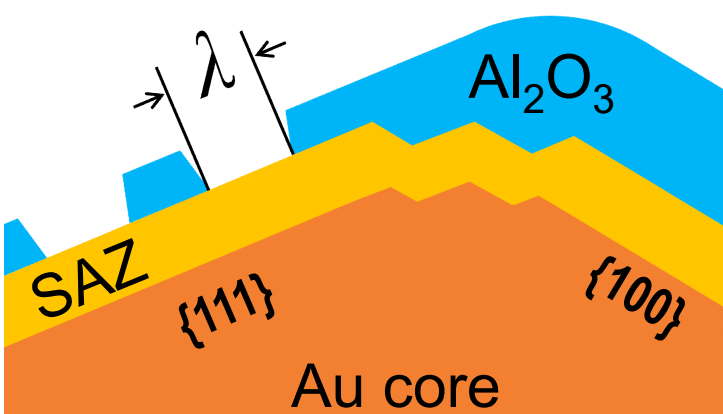

b)

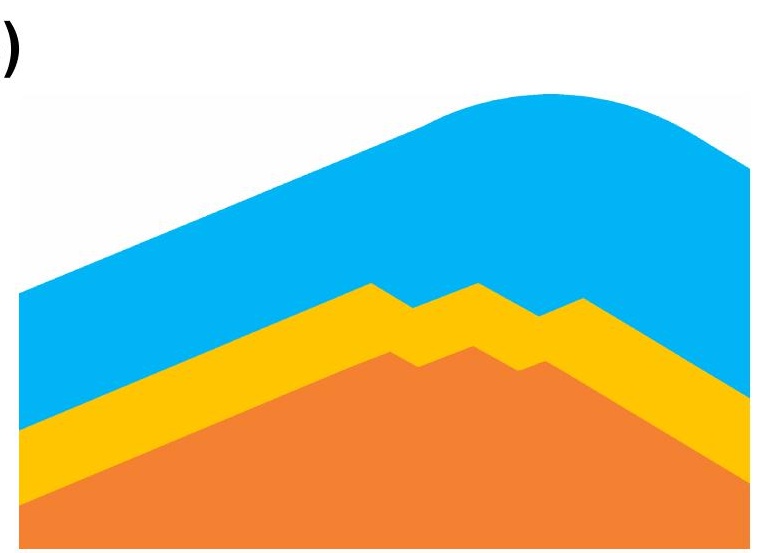




\section{a)}

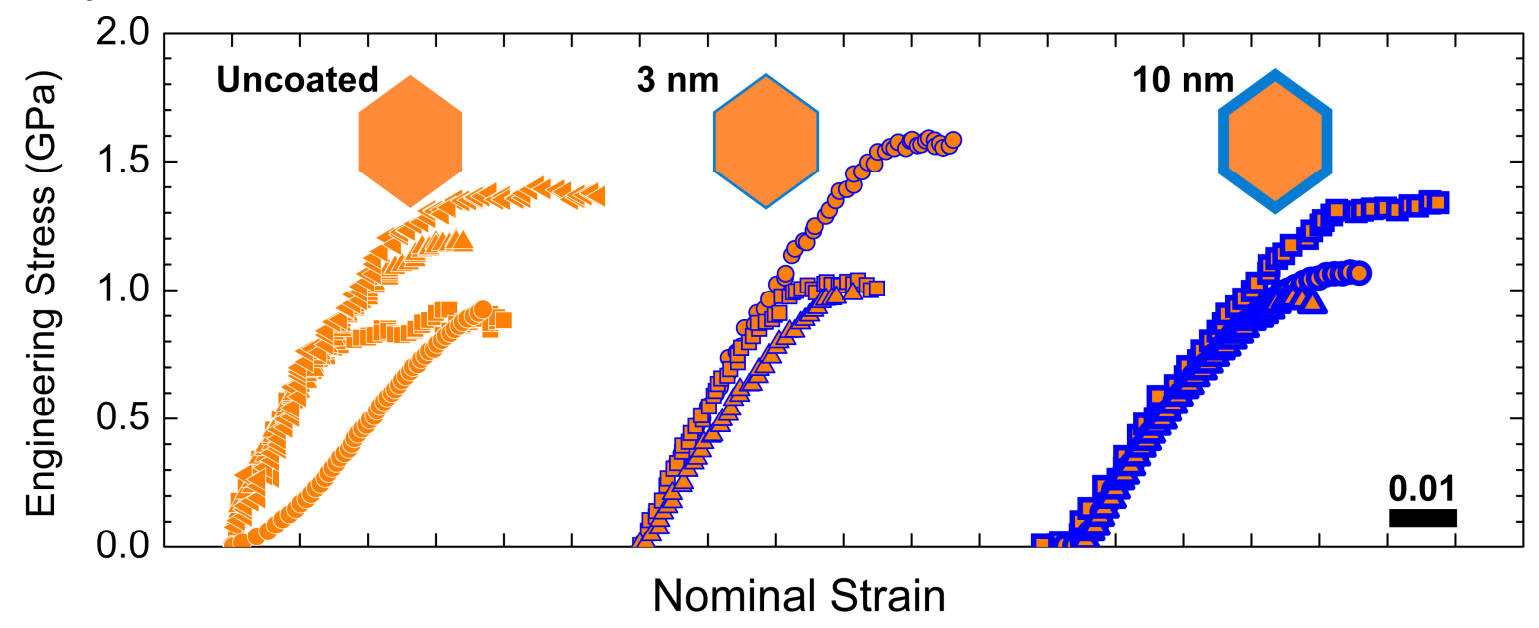

b)

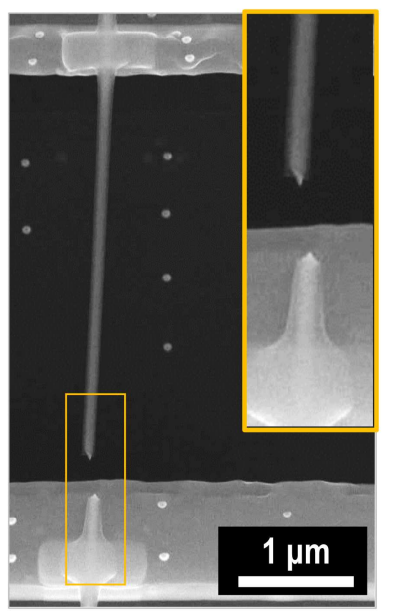

c)

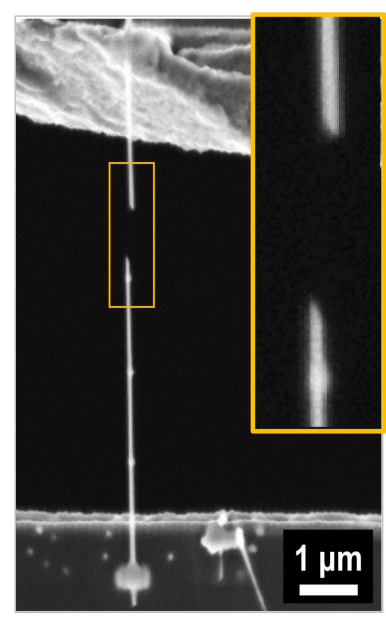

d)

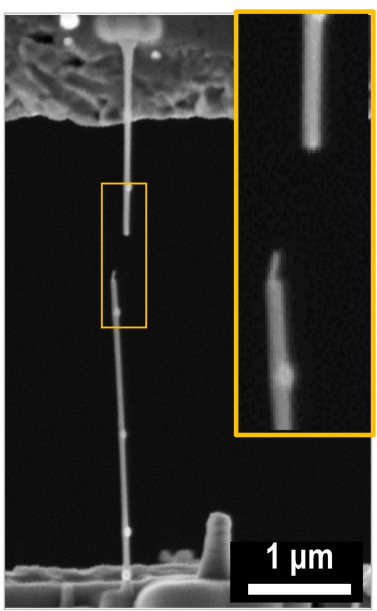




$$
\text { J1\% }
$$


a)

b)
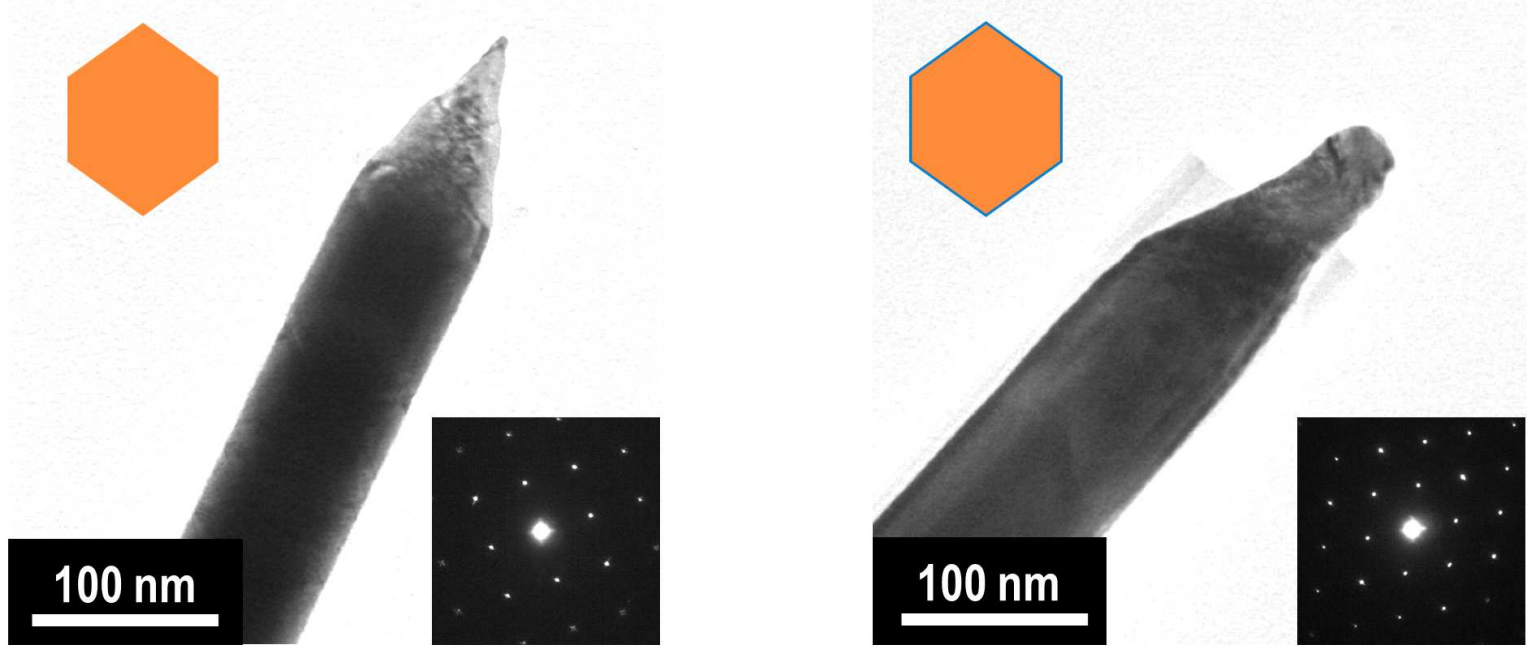

c)

d)
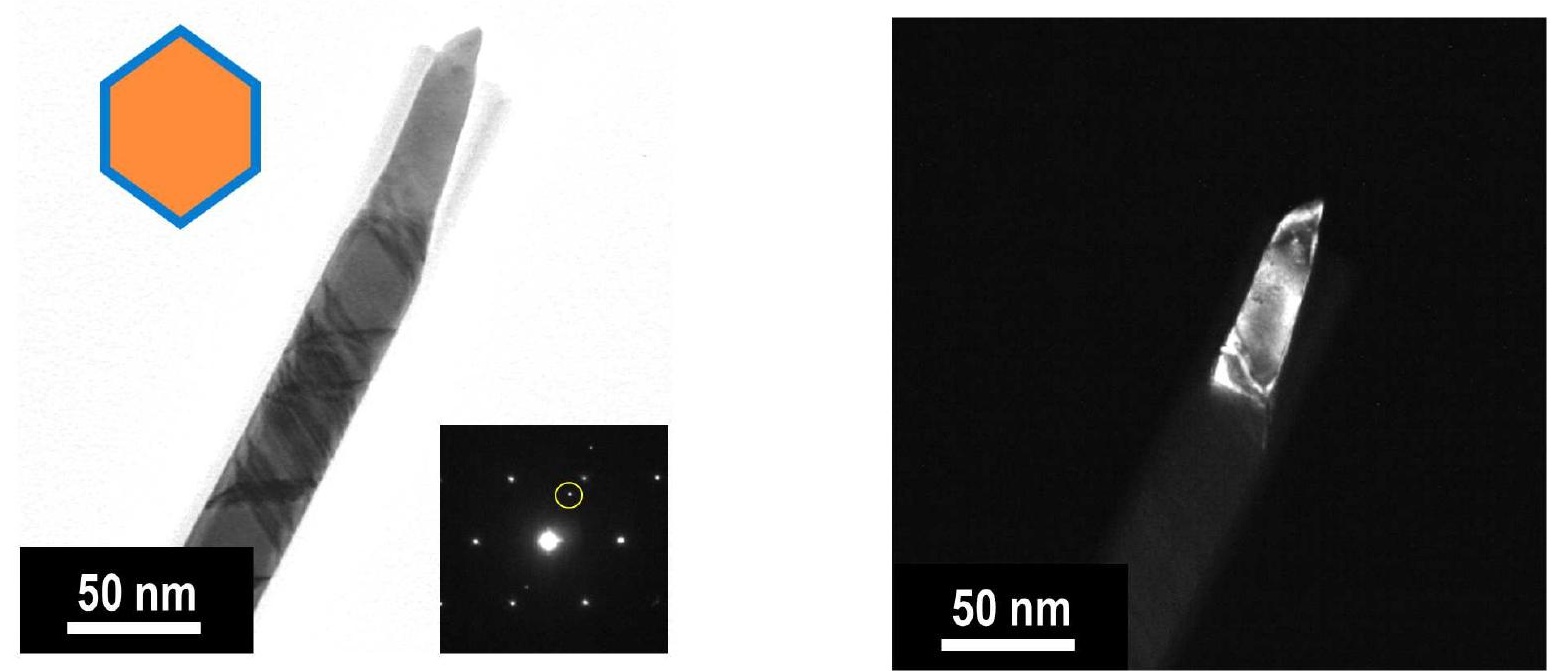
a)

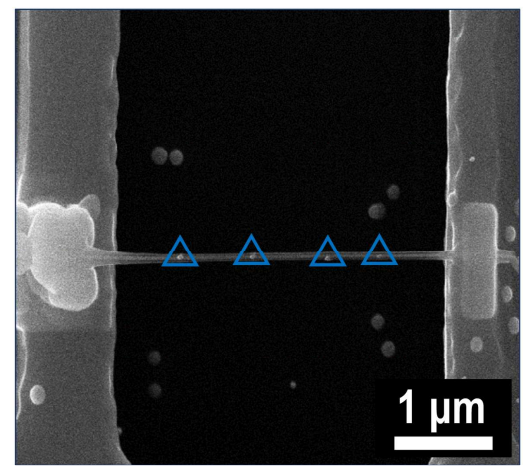

b)

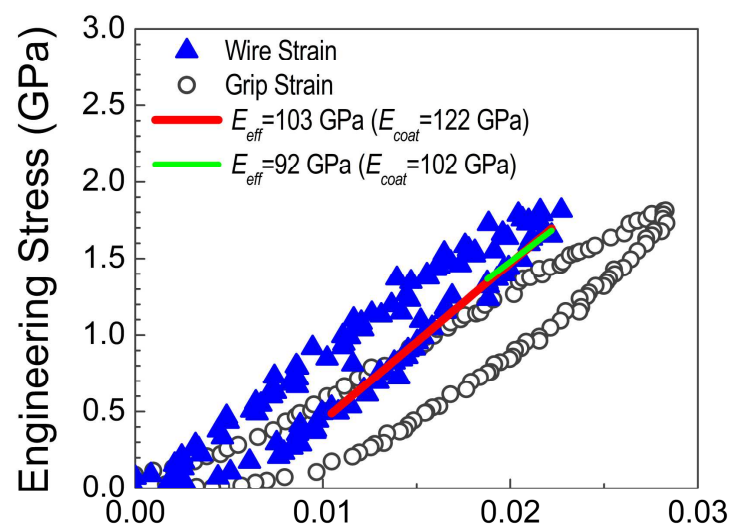

Engineering Strain 
ACCEPTED MANUSCRIPT

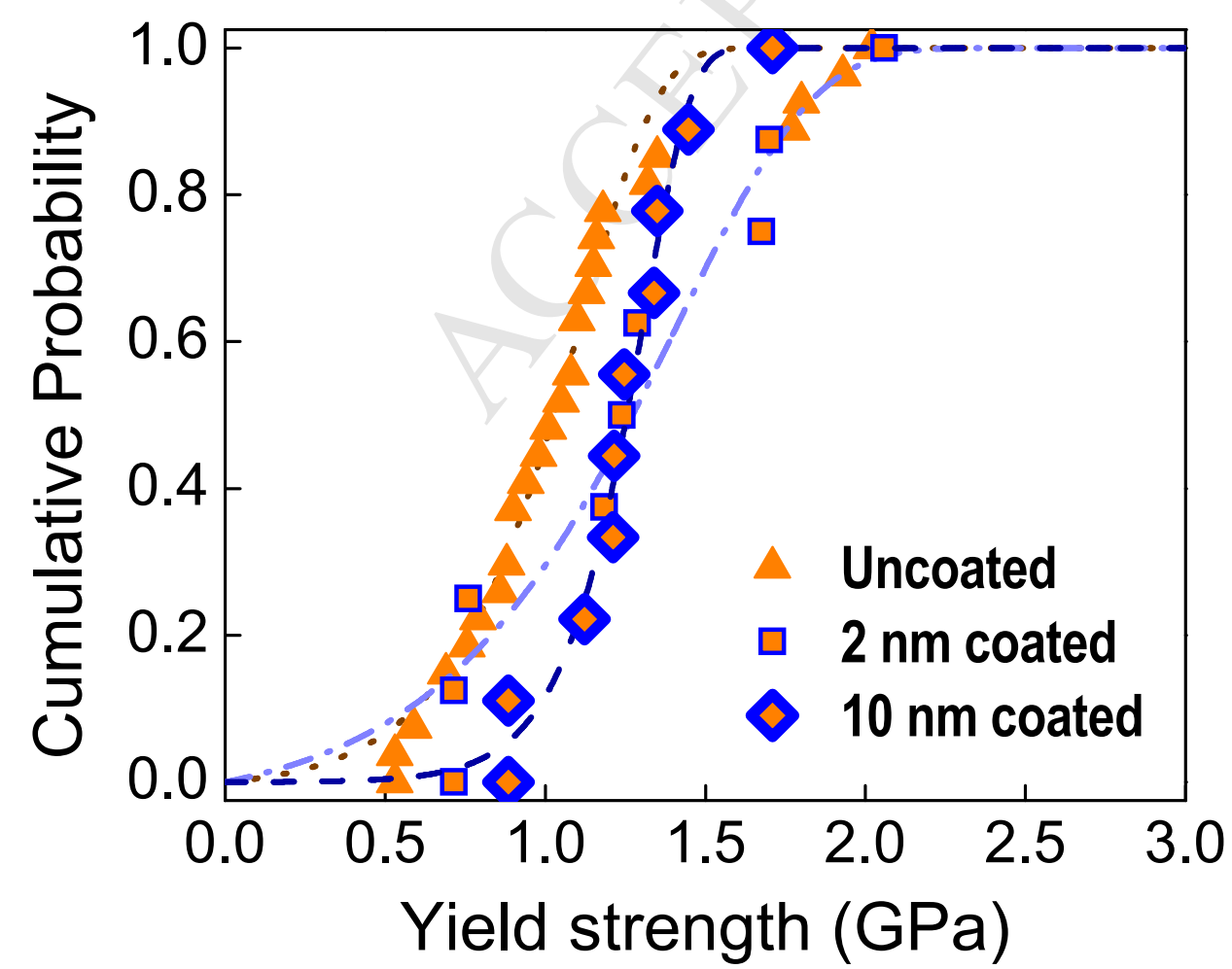


a)

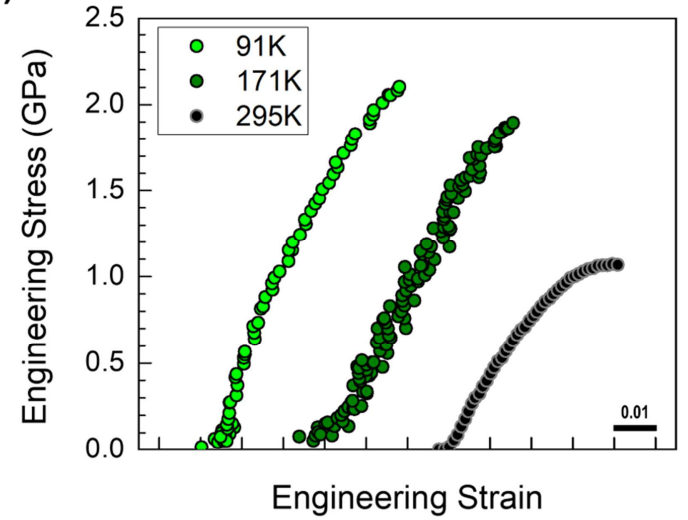

b)

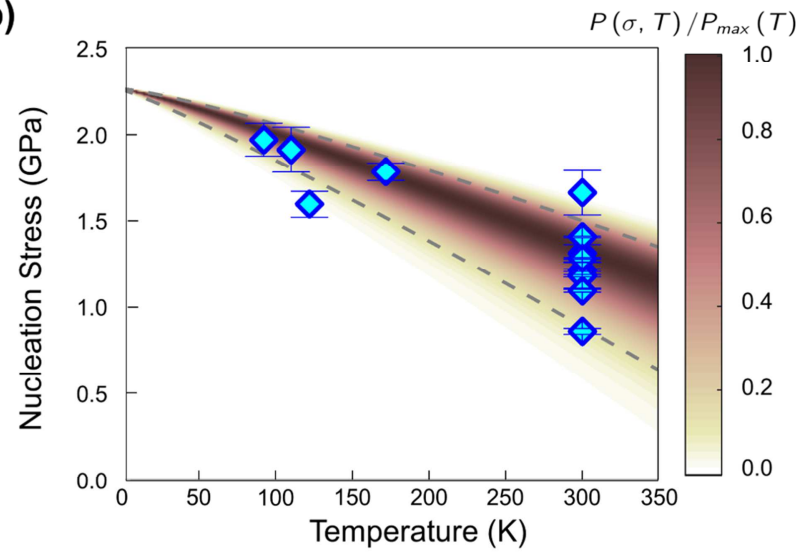

